\title{
Preparation of Alkenyl Cyclopropanes through a Ruthenium-Catalyzed Tandem Enyne Metathesis-Cyclopropanation Sequence
}

\author{
Byung Gyu Kim and Marc L. Snapper*
}

Department of Chemistry, Merkert Chemistry Center, Boston College, 2609 Beacon Street, Chestnut Hill, Massachusetts 024673860

\section{Supporting Information}

\section{Table of Contents}

(E)-2-[1-(Toluene-4-sulfonyl)-2,5-dihydro-1H-pyrrol-3-yl]-cyclopropanecarboxylic acid ethyl ester (4a) 


\section{General Information}

Unless otherwise stated, all reactions are carried out under an inert atmosphere $\mathrm{N}_{2}$ in flamed dried glassware. Starting material and reagents were purchased from chemical suppliers and used without further purification except the following: benzene and toluene were dried on alumina columns using a solvent dispensing system.

Infrared (IR) spectra are recorded on a Nicolet 210 spectrometer, $v_{\max }$ in $\mathrm{cm}^{-1} .{ }^{1} \mathrm{H}$ NMR spectra are recorded on a Varian Gemini-400 instrument $(400 \mathrm{MHz})$. Chemical shifts are reported in ppm from tetramethylsilane with the solvent resonance resulting from incomplete deuteration as the internal standard $\left(\mathrm{CDCl}_{3}: \delta\right.$ 7.26). Data are reported as follows: chemical shift, multiplicity ( $\mathrm{s}=$ singlet, $\mathrm{d}=$ doublet, $\mathrm{t}=$ triplet, $\mathrm{br}=$ broad, $\mathrm{m}=$ multiplet), coupling constant, and integration. ${ }^{13} \mathrm{C} N M R$ spectra are recorded on a Varian Gemini-400 instrument $(100 \mathrm{MHz})$ with complete proton decoupling. Chemical shifts are reported in ppm from tetramethylsilane with the solvent resonance as the internal standard $\left(\mathrm{CDCl}_{3}: \delta 77.00\right)$. High resolution mass spectrometry is performed by the University of Illinois Mass Spectrometry Laboratories (Urbana, Illinois).

\section{Experimental Procedures and Spectral Data}

Representative procedure for the synthesis of the tandem enyne ring closing metathesis and cyclopropanation: To a stirred solution of enyne (0.1-0.2 mmol, 1 equiv) in benzene (1-2 $\mathrm{mL})$ was added Grubbs' I catalyst 1 (0.01-0.02 mmol, 0.1 equiv) and degassed with ethylene for $5 \mathrm{~min}$. The reaction mixture was stirred for $10 \mathrm{~min}$ at $75{ }^{\circ} \mathrm{C}$ under ethylene atm. The reaction was then degassed with $\mathrm{N}_{2}$ for 5 min to remove residual ethylene. The reaction mixture was treated with diazo compound (0.5-1.0 mmol, 5 equiv.) in benzene (1.0-2.0 mL) over $10 \mathrm{~h}$ at $75^{\circ} \mathrm{C}$. After cooling to room temperature, the reaction was concentrated under the reduced pressure and the residue was purified by flash chromatography on silica gel to afford the $(E)$ - and $(Z)$-cyclopropyl stereoisomers.

Entry Enyne Diazo compound Product


(E)-2-[1-(Toluene-4-sulfonyl)-2,5-dihydro-1H-pyrrol-3-yl]-cyclopropanecarboxylic acid ethyl ester (4a) and (Z)-2-[1-(Toluene-4-sulfonyl)-2,5-dihydro-1H-pyrrol-3-yl]-cyclo-propanecarboxylic acid ethyl ester (4b). The reaction was conducted at $75{ }^{\circ} \mathrm{C}$. 5 equiv of ethyl diazoacetate and $10 \mathrm{~mol} \%$ of Grubbs' I catalyst 1 were used and the residue was purified by flash chromatography to afford Eisomer (4a) and Z-isomer (4b):

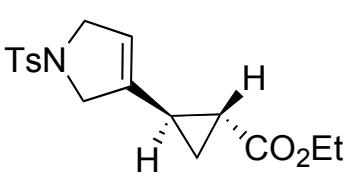

(4a) $45 \%$ yield, colorless oil. ${ }^{1} \mathbf{H}$ NMR $\left(400 \mathrm{MHz}, \mathrm{CDCl}_{3}\right) \delta 7.70$ (d, $J=8.0$ $\mathrm{Hz}, 2 \mathrm{H}), 7.32$ (d, $J=8.4 \mathrm{~Hz}, 2 \mathrm{H}), 5.34$ (bs, $1 \mathrm{H}), 4.11$ (q, $J=7.2 \mathrm{~Hz}, 2 \mathrm{H}$ ), 4.09-4.06 (m, 2H), 3.95-3.90 (m, 2H), $2.43(\mathrm{~s}, 3 \mathrm{H}), 1.95-1.90(\mathrm{~m}, 1 \mathrm{H}), 1.64$ $(\mathrm{dt}, J=9.6,4.8, \mathrm{~Hz}, 1 \mathrm{H}), 1.31(\mathrm{dt}, J=9.6,4.8 \mathrm{~Hz}, 1 \mathrm{H}), 1.25(\mathrm{t}, J=7.2 \mathrm{~Hz}$, $3 \mathrm{H}), 0.98$ (ddd, $J=9.8,7.2,4.8 \mathrm{~Hz}, 1 \mathrm{H}) .{ }^{13} \mathrm{C}$ NMR $\left(100 \mathrm{MHz}, \mathrm{CDCl}_{3}\right) \delta 172.6,143.3,137.6,134.0$, 129.6, 127.3, 119.0, 60.9, 55.1, 55.0, 21.6, 20.9, 20.4, 14.6, 14.3. IR (thin film, NaCl) $v_{\max } 2976,2902$, $2853,1732,1467,1417,1356,1196,1177,1103,814 \mathrm{~cm}^{-1}$. HRMS $(\mathrm{M}+\mathrm{H}) \mathrm{C}_{17} \mathrm{H}_{22} \mathrm{NO}_{4} \mathrm{~S}$ Calc. 336.1270 Obs. 336.1259

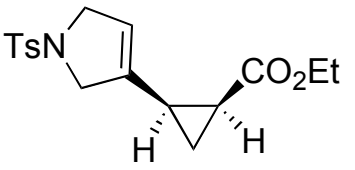

(4b). $20 \%$ yield, colorless oil. ${ }^{1} \mathrm{H}$ NMR $\left(400 \mathrm{MHz}, \mathrm{CDCl}_{3}\right) \delta 7.70(\mathrm{~d}, J=8.4$ $\mathrm{Hz}, 2 \mathrm{H}), 7.31(\mathrm{~d}, J=8.0 \mathrm{~Hz}, 2 \mathrm{H}), 5.40(\mathrm{~d}, J=1.6 \mathrm{~Hz}, 1 \mathrm{H}), 4.15-4.08(\mathrm{~m}$, $3 \mathrm{H}), 3.93(\mathrm{dq}, J=7.6,2.4 \mathrm{~Hz}, 2 \mathrm{H}), 3.89-3.84(\mathrm{~m}, 1 \mathrm{H}), 2.41(\mathrm{~s}, 3 \mathrm{H}), 1.87$ (ddd, $J=9.2,8.0,6.0 \mathrm{~Hz}, 1 \mathrm{H}), 1.75-1.69(\mathrm{~m}, 1 \mathrm{H}), 1.32(\mathrm{dt}, J=7.6,5.6 \mathrm{~Hz}, 1 \mathrm{H}), 1.14(\mathrm{td}, J=8.4$, $4.8 \mathrm{~Hz}, 1 \mathrm{H}), 1.11(\mathrm{t}, J=7.2 \mathrm{~Hz}, 3 \mathrm{H}) .{ }^{13} \mathrm{C}$ NMR $\left(100 \mathrm{MHz}, \mathrm{CDCl}_{3}\right) \delta 170.3,143.1,134.3,134.1$, 129.5, 127.3, 121.8, 60.5, 56.7, 55.0, 21.6, 20.7, 18.7, 14.3, 11.3. IR (thin film, NaCl) $v_{\max } 2992,2930$, $2844,1721,1414,1383,1297,1193,1168,1101,824 \mathrm{~cm}^{-1}$. HRMS $(\mathrm{M}+\mathrm{H}) \mathrm{C}_{17} \mathrm{H}_{22} \mathrm{NO}_{4} \mathrm{~S}$ Calc. 336.1270 Obs. 336.1264

(E)-2-[1-(Toluene-4-sulfonyl)-2,5-dihydro-1H-pyrrol-3-yl]-cyclo-propanecarboxylic acid tert-butyl ester (9a) and (Z)-2-[1-(Toluene-4-sulfonyl)-2,5-dihydro-1H-pyrrol-3-yl]-cyclo-propanecarboxylic acid tert-butyl ester (9b). The reaction was conducted at $75^{\circ} \mathrm{C} .5$ equiv of $t$-butyl diazoacetate and $10 \mathrm{~mol} \%$ of Grubbs' I catalyst 1 were used and the residue was purified by flash chromatography to afford E-isomer (9a) and Z-isomer (9b):

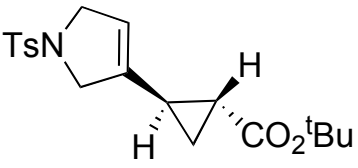

(9a). $52 \%$ yield, colorless oil. ${ }^{1} \mathrm{H}$ NMR $\left(400 \mathrm{MHz}, \mathrm{CDCl}_{3}\right) \delta 7.70(\mathrm{~d}, J=8.0$ $\mathrm{Hz}, 2 \mathrm{H}), 7.32(\mathrm{~d}, J=8.4 \mathrm{~Hz}, 2 \mathrm{H}), 5.32(\mathrm{dt}, J=2.0,0.8 \mathrm{~Hz}, 1 \mathrm{H}), 4.07-4.06$ $(\mathrm{m}, 2 \mathrm{H}), 3.95-3.91(\mathrm{~m}, 2 \mathrm{H}), 2.43(\mathrm{~s}, 3 \mathrm{H}), 1.88-1.83(\mathrm{~m}, 1 \mathrm{H}), 1.57$ (ddd, $J=$ 8.8, 5.6, $4.0 \mathrm{~Hz}, 1 \mathrm{H}$ ), 1.43 (s, 9H), 1.31 (ddd, $J=9.6,5.2,4.0 \mathrm{~Hz}, 1 \mathrm{H}$ ), 0.90 (ddd, $J=8.8,6.4,4.4$ $\mathrm{Hz}, 1 \mathrm{H}) .{ }^{13} \mathrm{C}$ NMR $\left(100 \mathrm{MHz}, \mathrm{CDCl}_{3}\right) \delta 171.7,143.3,137.9,134.2,129.6,127.3,118.7,80.9,55.1$, 
55.0, 28.2, 22.0, 21.6, 20.0, 14.4. IR (thin film, NaCl) $v_{\max } 2983,2934,2855,1729,1491,1461,1412$, 1376, 1364, 1254, 1224, 1163, 1114, $822 \mathrm{~cm}^{-1}$. HRMS $(\mathrm{M}+\mathrm{H}) \mathrm{C}_{19} \mathrm{H}_{26} \mathrm{NO}_{4} \mathrm{~S}$ Calc. 364.1583 Obs. 364.1569.

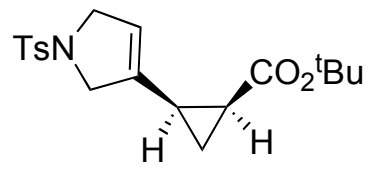

(9b). $19 \%$ yield, colorless oil. ${ }^{1} \mathrm{H}$ NMR $\left(400 \mathrm{MHz}, \mathrm{CDCl}_{3}\right) \delta 7.70$ (d, $J=8.4$ $\mathrm{Hz}, 2 \mathrm{H}), 7.29(\mathrm{~d}, J=8.0 \mathrm{~Hz}, 2 \mathrm{H}), 5.40(\mathrm{br} d, J=1.2 \mathrm{~Hz}, 1 \mathrm{H}), 4.14-4.03(\mathrm{~m}$, $3 \mathrm{H}), 3.91-3.86(\mathrm{~m}, 1 \mathrm{H}), 2.40(\mathrm{~s}, 3 \mathrm{H}), 1.80(\mathrm{ddd}, J=8.8,7.6,5.6 \mathrm{~Hz}, 1 \mathrm{H})$, 1.69-1.63 $(\mathrm{m}, 1 \mathrm{H}), 1.44-1.40(\mathrm{~m}, 1 \mathrm{H}), 1.26(\mathrm{~s}, 9 \mathrm{H}), 1.07(\mathrm{td}, J=8.4,5.2 \mathrm{~Hz}, 1 \mathrm{H}) .{ }^{13} \mathrm{C}$ NMR $(100$ $\left.\mathrm{MHz}, \mathrm{CDCl}_{3}\right) \delta 169.4,143.1,134.7,134.3,129.6,127.3,121.3,80.5,56.7,54.9,28.1,21.9,21.6$, 18.3, 10.8. IR (thin film, NaCl) $v_{\max }$ 2982, 2927, 2848, 1729, 1402, 1371, 1353, 1293, 1264, 1220, 1166, $1117,827 \mathrm{~cm}^{-1}$. HRMS $(\mathrm{M}+\mathrm{H}) \mathrm{C}_{19} \mathrm{H}_{26} \mathrm{NO}_{4} \mathrm{~S}$ Calc. 364.1583 Obs. 364.1567 .

2-[1-(Toluene-4-sulfonyl)-2,5-dihydro-1H-pyrrol-3-yl]-cyclo-propane-1,1-dicarboxylic acid dimethyl ester (10). The reaction was conducted at $100{ }^{\circ} \mathrm{C}$. 5 equiv of ethyl diazoacetate and $20 \mathrm{~mol} \%$ of Grubbs' I catalyst 1 were used and the residue was purified by flash chromatography to afford product.

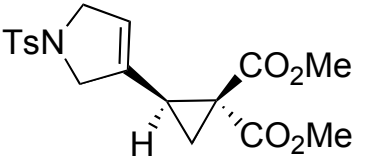

(10). $63 \%$, colorless oil. ${ }^{1} \mathrm{H}$ NMR $\left(400 \mathrm{MHz}, \mathrm{CDCl}_{3}\right) \delta 7.68(\mathrm{~d}, \mathrm{~J}=8.4 \mathrm{~Hz}$, $2 \mathrm{H}), 7.32(\mathrm{~d}, J=8.4 \mathrm{~Hz}, 2 \mathrm{H}), 5.31(\mathrm{q}, J=2.0 \mathrm{~Hz}, 1 \mathrm{H}), 4.13-4.08(\mathrm{~m}, 1 \mathrm{H})$, 4.06-3.99 (m, 3H), $3.72(\mathrm{~s}, 3 \mathrm{H}), 3.41(\mathrm{~s}, 3 \mathrm{H}), 2.41(\mathrm{~s}, 3 \mathrm{H}), 3.36(\mathrm{dd}, J=9.2$, $8.0 \mathrm{~Hz}, 1 \mathrm{H}), 1.73(\mathrm{dd}, J=8.0,5.2 \mathrm{~Hz}, 1 \mathrm{H}), 1.53(\mathrm{dd}, J=9.2,5.2 \mathrm{~Hz}, 1 \mathrm{H}) .{ }^{13} \mathrm{C} \mathbf{N M R}(100 \mathrm{MHz}$, $\left.\mathrm{CDCl}_{3}\right) \delta 169.4,166.6,143.3,133.7,129.6,127.4,121.6,56.2,54.9,53.0,52.5,36.5,25.4,21.6$, 18.6. IR (thin film, $\mathrm{NaCl}$ ) $v_{\max } 2959,2922,2849,1737,1437,1343,1304,1213,1196,1164,1129$, 1103, 1068, $817 \mathrm{~cm}^{-1}$. HRMS $\left(\mathrm{M}^{+}\right) \mathrm{C}_{18} \mathrm{H}_{21} \mathrm{NO}_{6} \mathrm{~S}$ Calc. 379.1090 Obs. 379.1082 .

(E)-1-(Toluene-4-sulfonyl)-3-(2-trimethylsilanyl-cyclopropyl)-2,5-dihydro-1H-pyrrole (11a) and (Z)-1(Toluene-4-sulfonyl)-3-(2-trimethylsilanyl-cyclopropyl)-2,5-dihydro-1H-pyrrole (11b). The reaction was conducted at $75{ }^{\circ} \mathrm{C}$. 5 equiv. of trimethylsilyl diazomethane $(2 \mathrm{M}$ in hexanes) and $10 \mathrm{~mol} \%$ of Grubbs' I catalyst 1 were used and the residue was purified by flash chromatography to afford $E$ isomer (11a) and Z-isomer (11b):

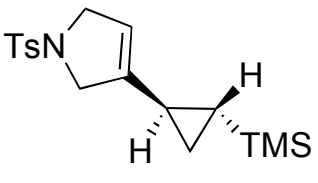

(11a). $26 \%$ yield, colorless oil. ${ }^{1} \mathrm{H}$ NMR $\left(400 \mathrm{MHz}, \mathrm{CDCl}_{3}\right) \delta 7.71$ (d, $J=8.4$ $\mathrm{Hz}, 2 \mathrm{H}), 7.32(\mathrm{~d}, J=8.4 \mathrm{~Hz}, 2 \mathrm{H}), 5.22-5.21(\mathrm{~m}, 1 \mathrm{H}), 4.07-4.05(\mathrm{~m}, 2 \mathrm{H}), 3.93-$ $3.89(\mathrm{~m}, 2 \mathrm{H}), 2.43(\mathrm{~s}, 3 \mathrm{H}), 1.22-1.18(\mathrm{~m}, 1 \mathrm{H}), 0.68$ (ddd, $J=10.0,4.4,4.0 \mathrm{~Hz}$, 
$1 \mathrm{H}), 0.56(\mathrm{td}, J=7.6,4.0 \mathrm{~Hz}, 1 \mathrm{H}),-0.07(\mathrm{~s}, 9 \mathrm{H}),-0.26(\mathrm{ddd}, J=10.4,7.6,6.4 \mathrm{~Hz}, 1 \mathrm{H}) .{ }^{13} \mathrm{C}$ NMR $\left(100 \mathrm{MHz}, \mathrm{CDCl}_{3}\right) \delta 143.1,141.6,134.3,129.6,127.3,115.9,55.1,55.0,21.6,14.2,9.7,6.3,-2.4$. IR (thin film, $\mathrm{NaCl}$ ) $v_{\max } 3004,2951,2891,2850,1654,1606,1458,1346,1248,1164,1102,925$, 837, $669 \mathrm{~cm}^{-1}$. HRMS $(\mathrm{M}+\mathrm{H}) \mathrm{C}_{17} \mathrm{H}_{26} \mathrm{NO}_{2} \mathrm{SSi}$ Calc. 336.1454 Obs. 336.1453 .

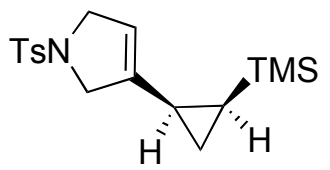

(11b). $8.5 \%$ yield, colorless oil. ${ }^{1} \mathrm{H}$ NMR $\left(400 \mathrm{MHz}, \mathrm{CDCl}_{3}\right) \delta 7.72(\mathrm{~d}, J=8.0$ $\mathrm{Hz}, 2 \mathrm{H}), 7.31(\mathrm{~d}, J=8.0 \mathrm{~Hz}, 2 \mathrm{H}), 5.12-5.11(\mathrm{~m}, 1 \mathrm{H}), 4.14-4.06(\mathrm{~m}, 2 \mathrm{H})$, 4.03-3.96 (m, 2H), $2.42(\mathrm{~s}, 3 \mathrm{H}), 1.42-1.36(\mathrm{~m}, 1 \mathrm{H}), 0.90$ (ddd, $J=10.0,8.0$, $4.4 \mathrm{~Hz}, 1 \mathrm{H}), 0.54$ (ddd, $J=8.0,5.6,4.0 \mathrm{~Hz}, 1 \mathrm{H}),-0.16(\mathrm{td}, J=10.4,8.0 \mathrm{~Hz}, 1 \mathrm{H}),-0.21(\mathrm{~s}, 9 \mathrm{H})$.

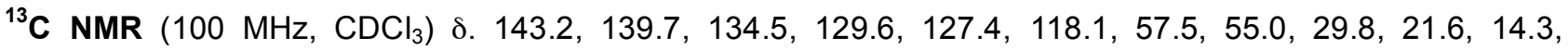
7.2, -0.7. IR (thin film, NaCl) $v_{\max } 2949,2925,2850,1458,1354,1249,1173,1115,1074,929,853$ $\mathrm{cm}^{-1}$. HRMS (M+H) $\mathrm{C}_{17} \mathrm{H}_{26} \mathrm{NO}_{2} \mathrm{SSi}$ Calc. 336.1454 Obs. 336.1462.

(E)-2-[1-(Toluene-4-sulfonyl)-1,2,5,6-tetroydro-1H-pyridin-3-yl]-cyclopropanecarboxylic acid ethyl ester (13a) and (Z)-2-[1-(Toluene-4-sulfonyl)-1,2,5,6-tetroydro-1H-pyridin-3-yl]-cyclopropanecarboxylic acid ethyl ester (13b). The reaction was conducted at $75{ }^{\circ} \mathrm{C}$. 5 equiv. of ethyl diazoacetate and $10 \mathrm{~mol} \%$ of Grubbs' I catalyst 1 were used and the residue was purified by flash chromatography to afford E-isomer (13a) and Z-isomer (13b):

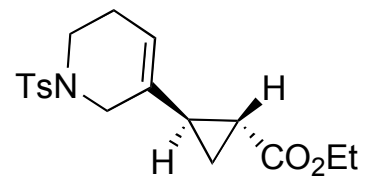

(13a). $48 \%$ yield, colorless oil. ${ }^{1} \mathrm{H}$ NMR $\left(400 \mathrm{MHz}, \mathrm{CDCl}_{3}\right) \delta 7.67(\mathrm{~d}, J=8.4$ $\mathrm{Hz}, 2 \mathrm{H}), 7.33(\mathrm{~d}, J=8.4 \mathrm{~Hz}, 2 \mathrm{H}), 5.49(\mathrm{~d}, J=1.6 \mathrm{~Hz}, 1 \mathrm{H}), 4.12(\mathrm{dq}, J=$ 7.2, $2.0 \mathrm{~Hz}, 2 \mathrm{H}), 3.47$ (bs, 2H), 3.13-3.06 (m, 2H), $2.43(\mathrm{~s}, 3 \mathrm{H}), 2.17$ (bs, $2 \mathrm{H}), 1.85-1.80(\mathrm{~m}, 1 \mathrm{H}), 1.59(\mathrm{dt}, J=9.2,4.4 \mathrm{~Hz}, 1 \mathrm{H}), 1.26(\mathrm{t}, J=7.2 \mathrm{~Hz}, 3 \mathrm{H}), 1.28-1.21(\mathrm{~m}, 1 \mathrm{H})$, 1.02 (ddd, $J=8.4,6.8,4.4 \mathrm{~Hz}, 1 \mathrm{H}) .{ }^{13} \mathrm{C}$ NMR $\left(100 \mathrm{MHz}, \mathrm{CDCl}_{3}\right) \delta 173.1,143.4,133.1,131.9,129.5$, 127.5, 119.6, 60.7, 46.8, 42.6, 25.2, 25.0, 21.6, 20.6, 14.3, 13.7. IR (thin film, NaCl) $v_{\max } 2991,2937$, $2848,1721,1466,1412,1341,1317,1211,1165,1094,973,825 \mathrm{~cm}^{-1}$. HRMS (M+H) $\mathrm{C}_{18} \mathrm{H}_{24} \mathrm{NO}_{4} \mathrm{~S}$ Calc. 350.1426 Obs. 350.1420 .

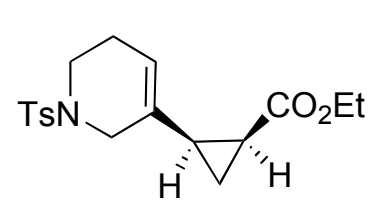

(13b). $20 \%$ yield, colorless oil. ${ }^{1} \mathrm{H}$ NMR $\left(400 \mathrm{MHz}, \mathrm{CDCl}_{3}\right) \delta 7.66$ (d, $J=8.4$ $\mathrm{Hz}, 2 \mathrm{H}), 7.31(\mathrm{~d}, J=8.4 \mathrm{~Hz}, 2 \mathrm{H}), 5.63(\mathrm{bs}, 1 \mathrm{H}), 3.95-3.87(\mathrm{~m}, 2 \mathrm{H}), 3.64(\mathrm{~d}$, $J=15.6 \mathrm{~Hz}, 1 \mathrm{H}), 3.28(\mathrm{dt}, J=11.2,5.6 \mathrm{~Hz}, 1 \mathrm{H}), 3.14(\mathrm{~d}, J=15.6 \mathrm{~Hz}, 1 \mathrm{H})$, 2.83 (ddd, $J=12.0,7.6,4.8 \mathrm{~Hz}, 1 \mathrm{H}), 2.42(\mathrm{~s}, 3 \mathrm{H}), 2.25-2.19(\mathrm{~m}, 2 \mathrm{H}), 1.82$ (ddd, $J=9.2,7.6,5.6 \mathrm{~Hz}, 1 \mathrm{H}), 1.80-1.75(\mathrm{~m}, 1 \mathrm{H}), 1.33(\mathrm{dt}, J=7.6,5.2 \mathrm{~Hz}, 1 \mathrm{H}), 1.09(\mathrm{t}, J=7.6$ $\mathrm{Hz}, 3 \mathrm{H}), 1.06(\mathrm{td}, J=8.0,4.8 \mathrm{~Hz}, 1 \mathrm{H}) .{ }^{13} \mathrm{C}$ NMR $\left(100 \mathrm{MHz}, \mathrm{CDCl}_{3}\right) \delta 170.6,143.2,133.4,129.4$, $128.8,127.6,123.0,60.4,47.7,42.6,25.3,24.3,21.6,19.9,14.4,10.2$. IR (thin film, NaCl) $v_{\max } 2971$, 
2916, 2862, 1730, 1458, 1379, 1355, 1161, 1101, $968 \mathrm{~cm}^{-1}$. HRMS (M+H) $\mathrm{C}_{18} \mathrm{H}_{24} \mathrm{NO}_{4} \mathrm{~S}$ Calc. 350.1426 Obs. 350.1418

(E)-2-[1-(Toluene-4-sulfonyl)-2,5,6,7-tetrahydro-1H-azepin-3-yl]-cyclopropanecarboxylic acid ethyl ester (15a) and (Z)-2-[1-(Toluene-4-sulfonyl)-2,5,6,7-tetrahydro-1H-azepin-3-yl]-cyclopropanecarboxylic acid ethyl ester (15b). The reaction was conducted at $75{ }^{\circ} \mathrm{C}$. 5 equiv of ethyl diazoacetate and $10 \mathrm{~mol} \%$ of Grubbs' I catalyst 1 were used and the residue was purified by flash chromatography to afford E-isomer (15a) and Z-isomer (15b):

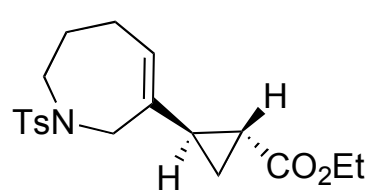

(15a). $35 \%$ yield, colorless oil. ${ }^{1} \mathrm{H}$ NMR $\left(400 \mathrm{MHz}, \mathrm{CDCl}_{3}\right) \delta 7.66(\mathrm{~d}, J=8.4$ $\mathrm{Hz}, 2 \mathrm{H}), 7.29(\mathrm{~d}, J=8.4 \mathrm{~Hz}, 2 \mathrm{H}), 5.58(\mathrm{t}, J=5.6 \mathrm{~Hz}, 1 \mathrm{H}), 4.14(\mathrm{q}, J=7.6$ $\mathrm{Hz}, 2 \mathrm{H}$ ), 3.70 (apparent dd, $J=21.2,16.8 \mathrm{~Hz}, 2 \mathrm{H}), 3.35(\mathrm{t}, J=6.4 \mathrm{~Hz}, 2 \mathrm{H}$ ), $2.42(\mathrm{~s}, 3 \mathrm{H}), 2.12(\mathrm{q}, J=5.6 \mathrm{~Hz}, 2 \mathrm{H}), 1.99-1.94(\mathrm{~m}, 1 \mathrm{H}), 1.78-1.70(\mathrm{~m}, 2 \mathrm{H})$, $1.68(\mathrm{dt}, J=8.4,4.8 \mathrm{~Hz}, 1 \mathrm{H}), 1.27(\mathrm{t}, J=7.6 \mathrm{~Hz}, 3 \mathrm{H}), 1.30-1.24(\mathrm{~m}, 1 \mathrm{H}), 1.08(\mathrm{ddd}, J=8.4,6.8$, $4.4 \mathrm{~Hz}, 1 \mathrm{H}) .{ }^{13} \mathrm{C}$ NMR $\left(100 \mathrm{MHz}, \mathrm{CDCl}_{3}\right) \delta 173.3,142.9,136.6,136.3,129.4,127.7,127.0,60.6$, 49.8, 48.4, 27.5, 26.8, 26.0, 21.6, 20.4, 14.4, 14.0. IR (thin film, NaCl) $v_{\max } 2982,2934,2842,1720$, 1467, 1424, 1333, 1272, 1193, 1159, 1096, 1041, $822 \mathrm{~cm}^{-1}$. HRMS (M+H) $\mathrm{C}_{19} \mathrm{H}_{26} \mathrm{NO}_{4} \mathrm{~S}$ Calc. 364.1583 Obs. 364.1577.

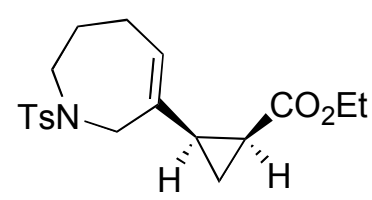

(15b). $18 \%$ yield, colorless oil. ${ }^{1} \mathrm{H}$ NMR $\left(400 \mathrm{MHz}, \mathrm{CDCl}_{3}\right) \delta 7.68$ (d, $J=8.4$ $\mathrm{Hz}, 2 \mathrm{H}), 7.29(\mathrm{~d}, J=8.0 \mathrm{~Hz}, 2 \mathrm{H}), 5.70(\mathrm{t}, J=5.6 \mathrm{~Hz}, 1 \mathrm{H}), 4.04(\mathrm{q}, J=7.2$ $\mathrm{Hz}, 2 \mathrm{H}), 3.94(\mathrm{~d}, J=16.8 \mathrm{~Hz}, 1 \mathrm{H}), 3.55(\mathrm{~d}, J=16.0 \mathrm{~Hz}, 1 \mathrm{H}), 3.49(\mathrm{q}, J=$ $6.0 \mathrm{~Hz}, 1 \mathrm{H}), 3.14(\mathrm{dt}, J=12.8,6.0 \mathrm{~Hz}, 1 \mathrm{H}), 2.42(\mathrm{~s}, 3 \mathrm{H}), 2.17(\mathrm{q}, J=4.0$ $\mathrm{Hz}, 2 \mathrm{H}), 2.01$ (q, $J=8.8 \mathrm{~Hz}, 1 \mathrm{H}), 1.83$ (ddd, $J=8.8,8.0,5.6 \mathrm{~Hz}, 1 \mathrm{H}), 1.77-1.71(\mathrm{~m}, 2 \mathrm{H}), 1.33$ (dt, $J=7.6,5.2 \mathrm{~Hz} 1 \mathrm{H}), 1.20(\mathrm{t}, J=7.2 \mathrm{~Hz}, 3 \mathrm{H}), 1.10(\mathrm{td}, J=8.4,5.2 \mathrm{~Hz}, 1 \mathrm{H}) .{ }^{13} \mathrm{C} \mathbf{N M R}(100 \mathrm{MHz}$, $\left.\mathrm{CDCl}_{3}\right) \delta 171.3,142.8,136.3,133.7,130.5,129.4,127.0,60.4,50.2,49.7,27.4,26.9,25.9,21.6$, 20.0, 14.4, 11.5. IR (thin film, NaCl) $v_{\max }$ 2983, 2934, 2849, 1723, 1456, 1390, 1336, 1195, 1159, 1093, 824, $722 \mathrm{~cm}^{-1}$. HRMS (M+H) $\mathrm{C}_{19} \mathrm{H}_{26} \mathrm{NO}_{4} \mathrm{~S}$ Calc. 364.1583 Obs. 364.1570 .

(E)-3-(2-Ethoxycarbonyl-cyclopropyl)-cyclopent-3-ene-1,1-dicarboxylic acid diethyl ester (17a) and (Z)-3-(2-Ethoxycarbonyl-cyclopropyl)-cyclopent-3-ene-1,1-dicarboxylic acid diethyl ester (17b). The reaction was conducted at $75{ }^{\circ} \mathrm{C}$. 5 equiv of ethyl diazoacetate and $10 \mathrm{~mol} \%$ of Grubbs' I catalyst 1 were used and the residue was purified by flash chromatography to afford E-isomer (17a) and Zisomer (17b): 


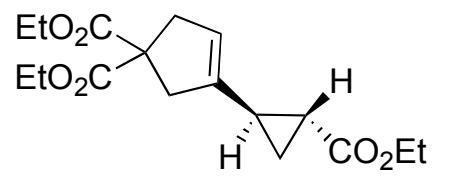

(17a). 38\% yield, colorless oil. ${ }^{1} \mathrm{H}$ NMR $\left(400 \mathrm{MHz}, \mathrm{CDCl}_{3}\right) \delta 5.34-5.33(\mathrm{~m}$, $1 \mathrm{H}), 4.19(\mathrm{q}, J=7.2 \mathrm{~Hz}, 2 \mathrm{H}), 4.18(\mathrm{q}, J=7.2 \mathrm{~Hz}, 2 \mathrm{H}), 4.15-4.08(\mathrm{~m}$, $2 \mathrm{H}), 2.97$ (bs, $2 \mathrm{H}), 2.81-2.80(\mathrm{~m}, 2 \mathrm{H}), 2.08-2.03(\mathrm{~m}, 1 \mathrm{H}), 1.72$ (ddd, $J=$ 8.4, 5.2, $4.0 \mathrm{~Hz}, 1 \mathrm{H}), 1.30$ (ddd, $J=9.2,5.2,4.4 \mathrm{~Hz}, 1 \mathrm{H}), 1.24(\mathrm{t}, J=7.2 \mathrm{~Hz}, 3 \mathrm{H}), 1.24(\mathrm{t}, J=7.2$ $\mathrm{Hz}, 3 \mathrm{H}), 1.24(\mathrm{t}, J=7.2 \mathrm{~Hz}, 3 \mathrm{H}), 1.06$ (ddd, $J=8.0,6.4,4.0 \mathrm{~Hz}, 1 \mathrm{H}) .{ }^{13} \mathrm{C}$ NMR $\left(100 \mathrm{MHz}, \mathrm{CDCl}_{3}\right)$ $\delta 173.3,171.7,139.5,121.7,61.6,60.7,58.9,40.8,40.5,22.6,20.6,14.5,14.4,14.2$. IR (thin film, $\mathrm{NaCl}) v_{\max } 2978,2942,2899,2863,1729,1454,1417,1387,1326,1251,1182,1077,1035,871 \mathrm{~cm}^{-1}$. HRMS $\left(\mathrm{M}^{+}\right) \mathrm{C}_{17} \mathrm{H}_{24} \mathrm{O}_{6}$ Calc. 324.1573 Obs. 324.1562 .

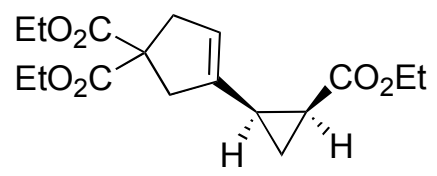

(17b). $37 \%$ yield, colorless oil. ${ }^{1} \mathrm{H}$ NMR $\left(400 \mathrm{MHz}, \mathrm{CDCl}_{3}\right) \delta 5.36-5.34(\mathrm{~m}$, $1 \mathrm{H}), 4.20-4.12(\mathrm{~m}, 4 \mathrm{H}), 4.10-4.02(\mathrm{~m}, 2 \mathrm{H}), 3.03-2.96(\mathrm{~m}, 3 \mathrm{H}), 2.86-2.81$

$(\mathrm{m}, 1 \mathrm{H}), 1.86(\mathrm{ddd}, J=9.2,7.6,6.0 \mathrm{~Hz}, 1 \mathrm{H}), 1.85-1.78(\mathrm{~m}, 1 \mathrm{H}), 1.38$ (ddd, $J=8.4,6.0,5.2 \mathrm{~Hz}, 1 \mathrm{H}), 1.23(\mathrm{t}, J=7.2 \mathrm{~Hz}, 3 \mathrm{H}), 1.22(\mathrm{t}, J=7.2 \mathrm{~Hz}, 3 \mathrm{H}), 1.21(\mathrm{t}, J=7.2$ $\mathrm{Hz}, 3 \mathrm{H}), 1.11$ (ddd, $J=8.4,7.6,5.2 \mathrm{~Hz}, 1 \mathrm{H}) .{ }^{13} \mathrm{C}$ NMR $\left(100 \mathrm{MHz}, \mathrm{CDCl}_{3}\right) \delta 171.9,171.6,170.7$, $136.3,124.4,61.5,61.4,60.4,59.2,43.3,40.4,29.8,20.8,20.7,14.4,14.2,11.4$. IR (thin film, $\mathrm{NaCl}$ ) $v_{\max } 2974,2937,2913,2869,1731,1474,1450,1381,1251,1181,1096,1069,10024,876,826 \mathrm{~cm}^{-1}$. HRMS (M) $\mathrm{C}_{17} \mathrm{H}_{24} \mathrm{O}_{6}$ Calc. 324.1573 Obs. 324.1559.

(E)-3-(2-Ethoxycarbonyl-cyclopropyl)-cyclopent-3-ene-1,1-dicarboxylic acid tert-butyl ester (18a) and (Z)-3-(2-Ethoxycarbonyl-cyclopropyl)-cyclopent-3-ene-1,1-dicarboxylic acid tert-butyl ester (18b). The reaction was conducted at $75^{\circ} \mathrm{C} .5$ equiv of $t$-butyl diazoacetate and $10 \mathrm{~mol} \%$ of Grubbs' I catalyst 1 were used and the residue was purified by flash chromatography to afford $E$-isomer (18a) and Z-isomer (18b):

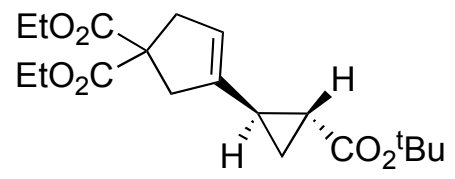

(18a). 37\% yield, colorless oil. ${ }^{1} \mathbf{H}$ NMR $\left(400 \mathrm{MHz}, \mathrm{CDCl}_{3}\right) \delta$ 5.33-5.32 $(\mathrm{m}, 1 \mathrm{H}), 4.19$ (q, $J=7.2 \mathrm{~Hz}, 2 \mathrm{H}), 4.18(\mathrm{q}, J=7.2 \mathrm{~Hz}, 2 \mathrm{H}), 2.99-2.97$ $(\mathrm{m}, 2 \mathrm{H}), 2.84-2.79(\mathrm{~m}, 2 \mathrm{H}), 2.02-1.97(\mathrm{~m}, 1 \mathrm{H}), 1.64(\mathrm{ddd}, J=8.4,5.2$, $4.4 \mathrm{~Hz}, 1 \mathrm{H}), 1.44(\mathrm{~s}, 9 \mathrm{H}), 1.24(\mathrm{t}, J=7.2 \mathrm{~Hz}, 3 \mathrm{H}), 1.24(\mathrm{t}, J=7.2 \mathrm{~Hz}, 3 \mathrm{H}), 1.22$ (ddd, $J=9.2,5.2$, $4.4 \mathrm{~Hz}, 1 \mathrm{H}), 0.99$ (ddd, $J=8.4,6.0,4.4 \mathrm{~Hz}, 1 \mathrm{H}) .{ }^{13} \mathrm{C}$ NMR $\left(100 \mathrm{MHz}, \mathrm{CDCl}_{3}\right) \delta 172.4,171.7,139.7$, $121.4,80.4,61.6,58.8,40.8,40.5,28.2,22.1,21.5,14.3,14.1$. IR (thin film, NaCl) $v_{\max } 2979,2942$, 2867, 1731, 1444, 1402, 1367, 1325, 1251, 1214, 1153, 1096, 1071, 1016, $853 \mathrm{~cm}^{-1}$. LRMS (M+) $\mathrm{C}_{19} \mathrm{H}_{28} \mathrm{O}_{6}$ Calc. 352.2 Obs. 352.2. HRMS (M+H) $\mathrm{C}_{19} \mathrm{H}_{29} \mathrm{O}_{6}$ Calc. 353.1964 Obs. 353.1970. 


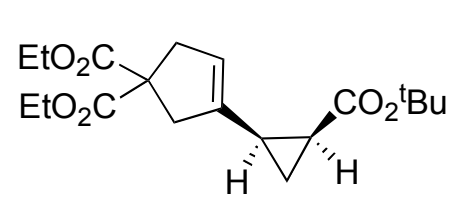

(18b). $35 \%$ yield, colorless oil. ${ }^{1} \mathbf{H}$ NMR $\left(400 \mathrm{MHz}, \mathrm{CDCl}_{3}\right) \delta 5.34-5.33$ $(\mathrm{m}, 1 \mathrm{H}), 4.18(\mathrm{q}, J=7.2 \mathrm{~Hz}, 2 \mathrm{H}), 4.15(\mathrm{q}, J=7.2 \mathrm{~Hz}, 2 \mathrm{H}), 3.07-3.02$ $(\mathrm{m}, 1 \mathrm{H}), 2.99(\mathrm{t}, J=2.0 \mathrm{~Hz}, 2 \mathrm{H}), 2.88-2.82(\mathrm{~m}, 1 \mathrm{H}), 1.78(\mathrm{dd}, J=7.6$, $6.0 \mathrm{~Hz}, 1 \mathrm{H}), 1.78-1.75(\mathrm{~m}, 1 \mathrm{H}), 1.39(\mathrm{~s}, 9 \mathrm{H}), 1.31(\mathrm{ddd}, J=6.8,5.6$, $4.4 \mathrm{~Hz}, 1 \mathrm{H}), 1.24(\mathrm{t}, J=7.2 \mathrm{~Hz}, 3 \mathrm{H}), 1.22(\mathrm{t}, J=7.2 \mathrm{~Hz}, 3 \mathrm{H}), 1.06(\mathrm{td}, J=8.4,4.8 \mathrm{~Hz}, 1 \mathrm{H}) .{ }^{13} \mathrm{C}$ NMR $\left(100 \mathrm{MHz}, \mathrm{CDCl}_{3}\right) \delta 172.0,171.6,169.9,136.5,123.9,80.2,61.5,61.4,59.2,43.5,40.4,29.8$, 28.2, 21.9, 20.6, 14.1, 11.1. IR (thin film, NaCl) $v_{\max } 2977,2940,2873,1731,1447,1391,1366,1250$, 1215, 1158, 1143, 1099, 1068, $1025 \mathrm{~cm}^{-1}$. LRMS $\left(\mathrm{M}^{+}\right) \mathrm{C}_{19} \mathrm{H}_{28} \mathrm{O}_{6}$ Calc. 352.2 Obs. 352.2. HRMS $(\mathrm{M}+\mathrm{H}) \mathrm{C}_{19} \mathrm{H}_{29} \mathrm{O}_{6}$ Calc. 353.1964 Obs. 353.1961.

(E)-3-(2-Ethoxycarbonyl-cyclopropyl)-cyclohex-3-ene-1,1-dicarboxylic acid diethyl ester (20a) and (Z)-3-(2-Ethoxycarbonyl-cyclopropyl)-cyclohex-3-ene-1,1-dicarboxylic acid diethyl ester (20b). The reaction was conducted at $75{ }^{\circ} \mathrm{C}$. 5 equiv of ethyl diazoacetate and $10 \mathrm{~mol} \%$ of Grubbs' I catalyst 1 were used and the residue was purified by flash chromatography to afford E-isomer (20a) and Zisomer (20b):

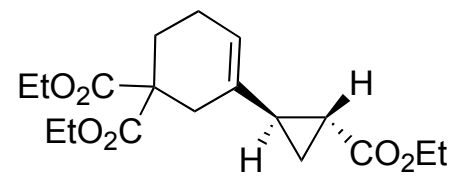

(20a). $44 \%$ yield, colorless oil. ${ }^{1} \mathrm{H}$ NMR $\left(400 \mathrm{MHz}, \mathrm{CDCl}_{3}\right) \delta 5.48$ (br d, $J=1.2 \mathrm{~Hz}, 1 \mathrm{H}), 4.18(\mathrm{q}, J=7.2 \mathrm{~Hz}, 2 \mathrm{H}), 4.18(\mathrm{q}, J=7.2 \mathrm{~Hz}, 2 \mathrm{H})$, 4.11 (dq, $J=7.2,4.0 \mathrm{~Hz}, 2 \mathrm{H}$ ), 2.37 (bs, 2H), 2.08 (bs, 4H), 2.01-1.96 $(\mathrm{m}, 1 \mathrm{H}), 1.68$ (ddd, $J=8.8,4.8,4.4 \mathrm{~Hz}, 1 \mathrm{H}), 1.28-1.21(\mathrm{~m}, 10 \mathrm{H}), 1.09$ (ddd, $J=11.2,8.8,6.0 \mathrm{~Hz}$, $1 \mathrm{H}) .{ }^{13} \mathrm{C}$ NMR $\left(100 \mathrm{MHz}, \mathrm{CDCl}_{3}\right) \delta 173.6,171.0,132.6,121.1,61.4,60.5,53.4,32.0,28.1,27.5$, 22.6, 19.7, 14.4, 14.2, 13.4. IR (thin film, NaCl) $v_{\max } 2977,2934,2897,2847,1738,1459,1410,1379$, 1311, 1255, 1193, 1100, 1044, $865 \mathrm{~cm}^{-1}$. HRMS $(\mathrm{M}+\mathrm{H}) \mathrm{C}_{18} \mathrm{H}_{27} \mathrm{O}_{6}$ Calc. 339.1808 Obs. 339.1792.

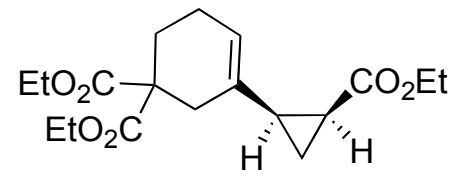

(20b). $25 \%$ yield, colorless oil. ${ }^{1} \mathrm{H}$ NMR $\left(400 \mathrm{MHz}, \mathrm{CDCl}_{3}\right) \delta 5.52$ (bs, $1 \mathrm{H}), 4.20-4.13(\mathrm{~m}, 4 \mathrm{H}), 4.12-4.04(\mathrm{~m}, 2 \mathrm{H}), 2.62(\mathrm{~d}, J=16.8 \mathrm{~Hz}, 1 \mathrm{H})$, $2.24(\mathrm{~d}, J=16.8 \mathrm{~Hz}, 1 \mathrm{H}), 2.15-2.07(\mathrm{~m}, 3 \mathrm{H}), 1.93(\mathrm{td}, J=12.8,5.6 \mathrm{~Hz}$, $1 \mathrm{H}), 1.83(\mathrm{t}, J=7.2 \mathrm{~Hz}, 2 \mathrm{H}), 1.37(\mathrm{q}, J=6.4 \mathrm{~Hz}, 1 \mathrm{H}), 1.25-1.18(\mathrm{~m}, 9 \mathrm{H}), 1.03$ (ddd, $J=12.8,8.0$, $1.2 \mathrm{~Hz}, 1 \mathrm{H}) .{ }^{13} \mathrm{C}$ NMR $\left(100 \mathrm{MHz}, \mathrm{CDCl}_{3}\right) \delta 171.3,171.0,129.5,123.3,61.3,61.2,60.3,53.6,34.4$, 27.3, 27.1, 22.5, 20.1, 14.5, 14.2, 10.5. IR (thin film, NaCl) $v_{\max } 2988,2927,2897,2854,1734,1455$, $1260,1187,1083,1059,1022,864 \mathrm{~cm}^{-1}$. HRMS $(\mathrm{M}+\mathrm{H}) \mathrm{C}_{18} \mathrm{H}_{27} \mathrm{O}_{6}$ Calc. 339.1808 Obs. 339.1793.

(E)-3-(2-Ethoxycarbonyl-1-methyl-cyclopropyl)-cyclopent-3-ene-1,1-dicarboxylic acid tert-butyl ester (22a) and (Z)-3-(2-Ethoxycarbonyl-1-methyl-cyclopropyl)-cyclopent-3-ene-1,1-dicarboxylic acid tertbutyl ester (22b). The reaction was conducted at $100{ }^{\circ} \mathrm{C}$. 5 equiv. of $t$-butyl diazoacetate and 20 
mol\% of Grubbs' I catalyst 1 were used and the residue was purified by flash chromatography to afford E-isomer (22a) and Z-isomer (22b):

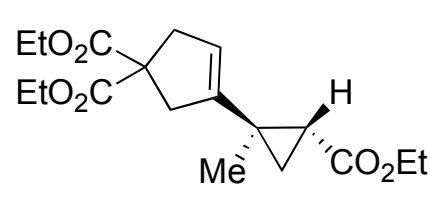

(22a). 32\% yield, colorless oil. ${ }^{1} \mathbf{H}$ NMR $\left(400 \mathrm{MHz}, \mathrm{CDCl}_{3}\right) \delta 5.35-5.34$ (m, $1 \mathrm{H}), 4.18(\mathrm{q}, J=7.2 \mathrm{~Hz}, 2 \mathrm{H}), 4.16(\mathrm{q}, J=7.2 \mathrm{~Hz}, 2 \mathrm{H}), 4.04$ (dq, $J=$ 7.2, $2.0 \mathrm{~Hz}, 2 \mathrm{H}), 3.09(\mathrm{dq}, J=16.8,2.0 \mathrm{~Hz}, 1 \mathrm{H}), 2.97(\mathrm{dq}, J=6.0,2.0$ $\mathrm{Hz}, 2 \mathrm{H}), 2.73(\mathrm{dq}, J=16.8,2.0 \mathrm{~Hz}, 1 \mathrm{H}), 1.65(\mathrm{dd}, J=8.0,6.0 \mathrm{~Hz}, 1 \mathrm{H})$, $1.52(\mathrm{dd}, J=6.0,4.8 \mathrm{~Hz}, 1 \mathrm{H}), 1.27(\mathrm{~s}, 3 \mathrm{H}), 1.24(\mathrm{t}, J=6.8 \mathrm{~Hz}, 3 \mathrm{H}), 1.23(\mathrm{t}, J=6.8 \mathrm{~Hz}, 3 \mathrm{H}), 1.21$ $(\mathrm{t}, J=6.8 \mathrm{~Hz}, 3 \mathrm{H}), 0.95(\mathrm{dd}, J=8.0,4.8 \mathrm{~Hz}, 1 \mathrm{H}) \cdot{ }^{13} \mathrm{C} \mathbf{N M R}\left(100 \mathrm{MHz}, \mathrm{CDCl}_{3}\right) \delta 171.9,171.5$, 170.6, 140.1, 123.7, 61.5, 61.4, 60.3, 41.4, 40.1, 28.6, 28.3, 26.9, 25.8, 24.7, 19.7, 14.4, 14.2. IR (thin film, NaCl) $v_{\max } 2990,2919,2865,1743,1457,1379,1254,1176,1069,1027,866 \mathrm{~cm}^{-1}$. HRMS $\left(\mathrm{M}^{+}\right) \mathrm{C}_{18} \mathrm{H}_{26} \mathrm{O}_{6}$ Calc. 338.1729 Obs. 338.1730.

(22b). $30 \%$ yield, colorless oil. ${ }^{1} \mathbf{H}$ NMR $\left(400 \mathrm{MHz}, \mathrm{CDCl}_{3}\right) \delta 5.36-5.35(\mathrm{~m}, 1 \mathrm{H}), 4.19$ (q, J=7.2 Hz,

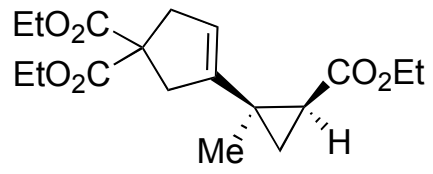
$2 \mathrm{H}), 4.19(\mathrm{q}, J=7.2 \mathrm{~Hz}, 2 \mathrm{H}), 4.15-4.11(\mathrm{~m}, 2 \mathrm{H}), 3.00(\mathrm{q}, J=2.0 \mathrm{~Hz}$, $2 \mathrm{H}), 2.83(\mathrm{q}, J=2.0 \mathrm{~Hz}, 1 \mathrm{H}), 2.82(\mathrm{q}, J=2.0 \mathrm{~Hz}, 1 \mathrm{H}), 1.84(\mathrm{dd}, J=$ 8.0, $6.4 \mathrm{~Hz}, 1 \mathrm{H}), 1.33(\mathrm{~s}, 3 \mathrm{H}), 1.26(\mathrm{t}, J=7.2 \mathrm{~Hz}, 3 \mathrm{H}), 1.25(\mathrm{t}, J=7.2$ $\mathrm{Hz}, 3 \mathrm{H}), 1.25(\mathrm{t}, J=7.2 \mathrm{~Hz}, 3 \mathrm{H}), 1.29-1.21(\mathrm{~m}, 2 \mathrm{H}) .{ }^{13} \mathrm{C} \mathrm{NMR}\left(100 \mathrm{MHz}, \mathrm{CDCl}_{3}\right) \delta 171.7,129.0$, $121.0,61.6,60.5,40.4,40.1,33.2,26.5,25.9,19.7,16.4,14.5,14.2$. IR (thin film, NaCl) $v_{\max } 2984$, 2936, 1739, 1466, 1411, 1393, 1265, 1192, 1071, 1016, $864 \mathrm{~cm}^{-1}$. HRMS $\left(\mathrm{M}^{+}\right) \mathrm{C}_{18} \mathrm{H}_{26} \mathrm{O}_{6}$ Calc. 338.1729 Obs. 338.1746 . 
${ }^{1} \mathrm{H}$ NMR of $4 a$

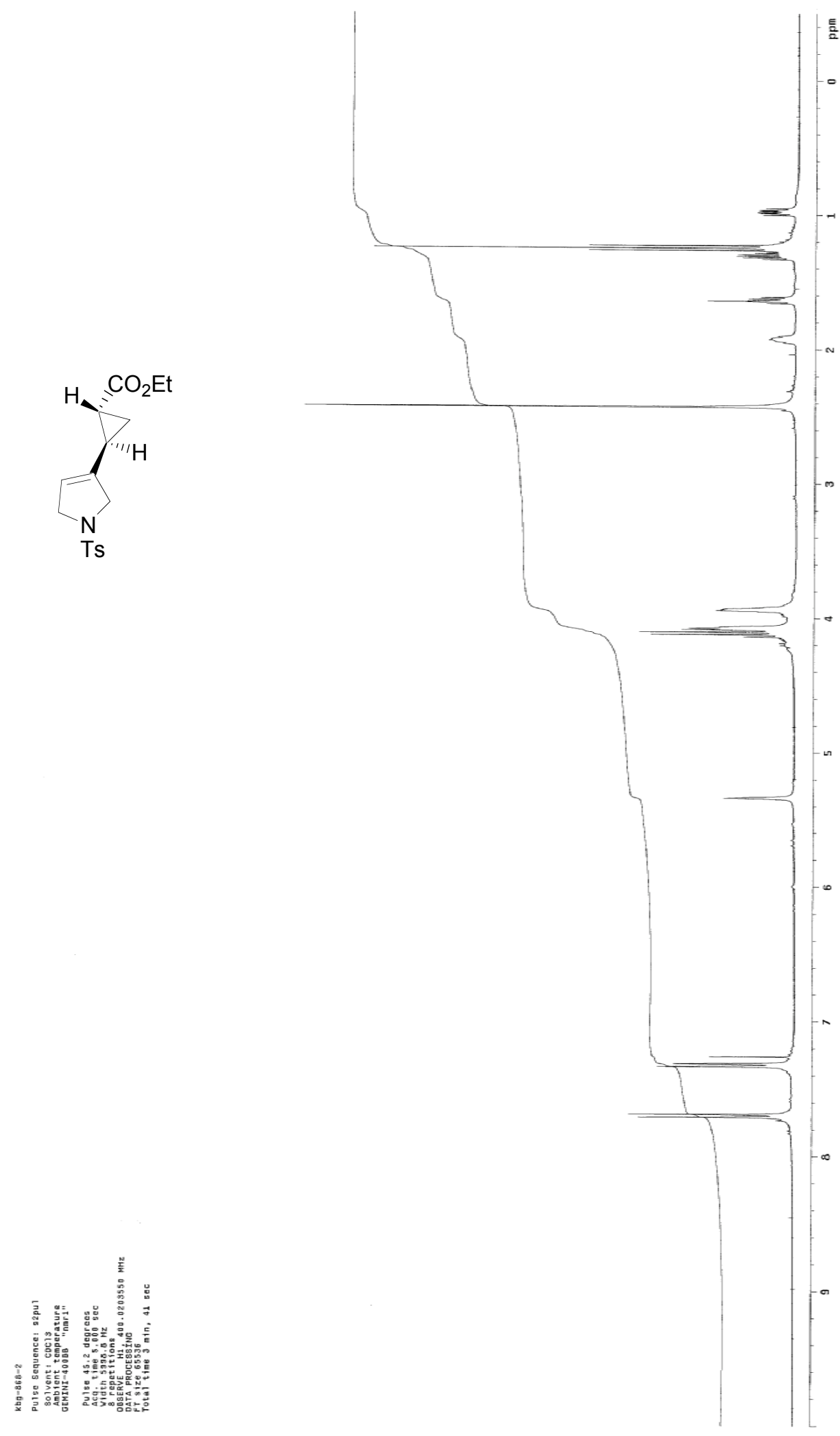


${ }^{1} \mathrm{H}$ NMR of $\mathbf{4 b}$

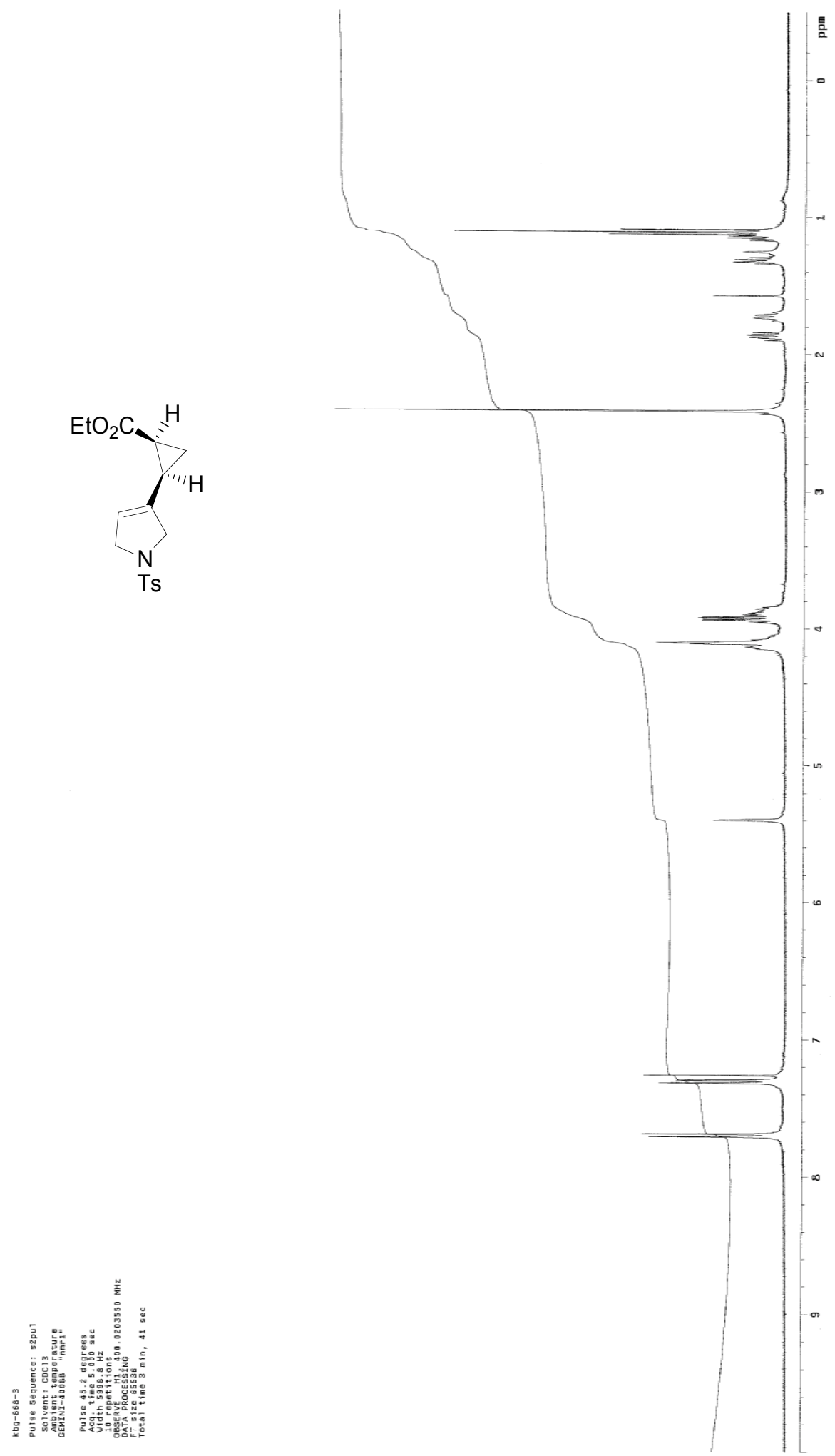


${ }^{1} \mathrm{H}$ NMR of $9 \mathbf{a}$

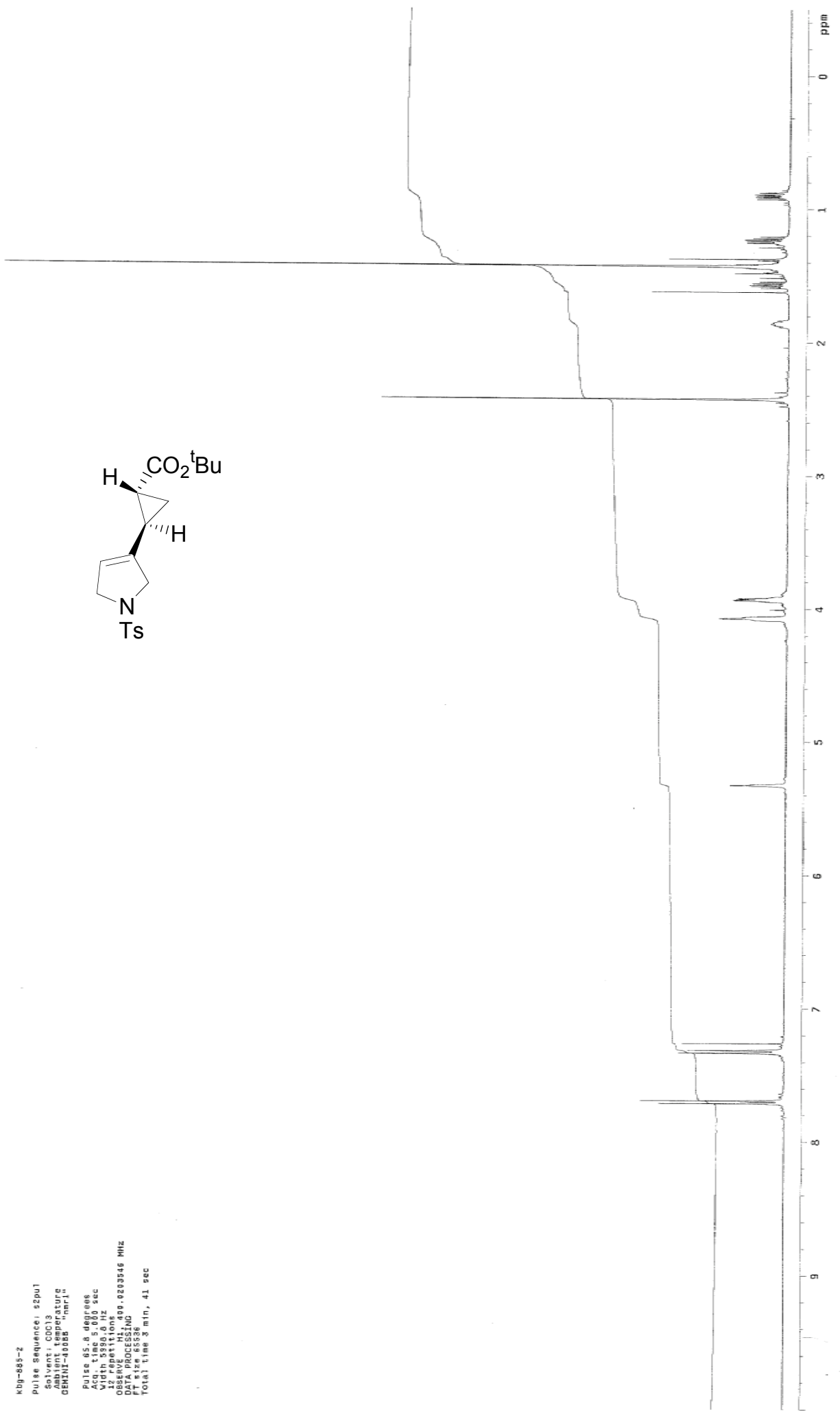


${ }^{1} \mathrm{H}$ NMR of $9 b$

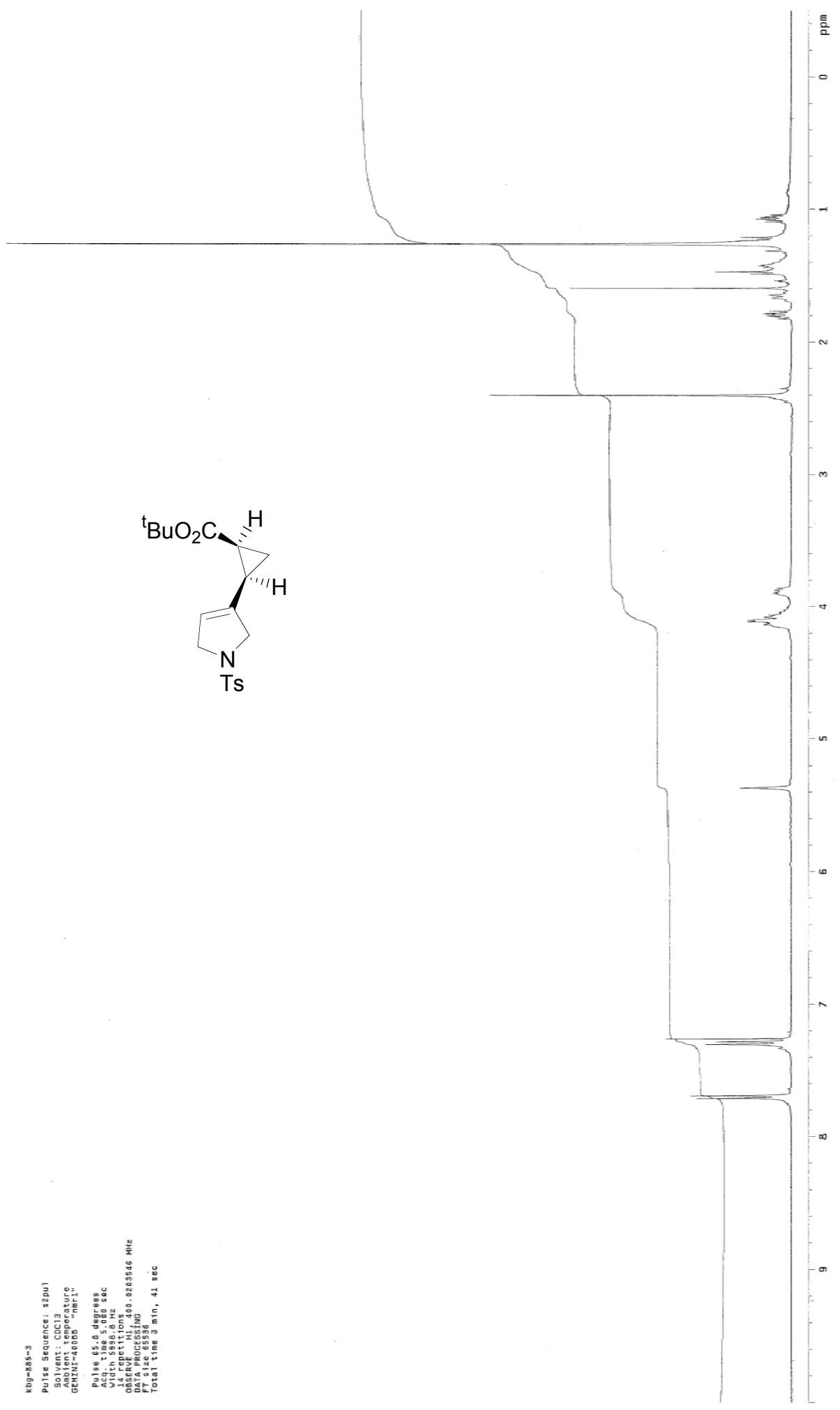


${ }^{1} \mathrm{H}$ NMR of 10
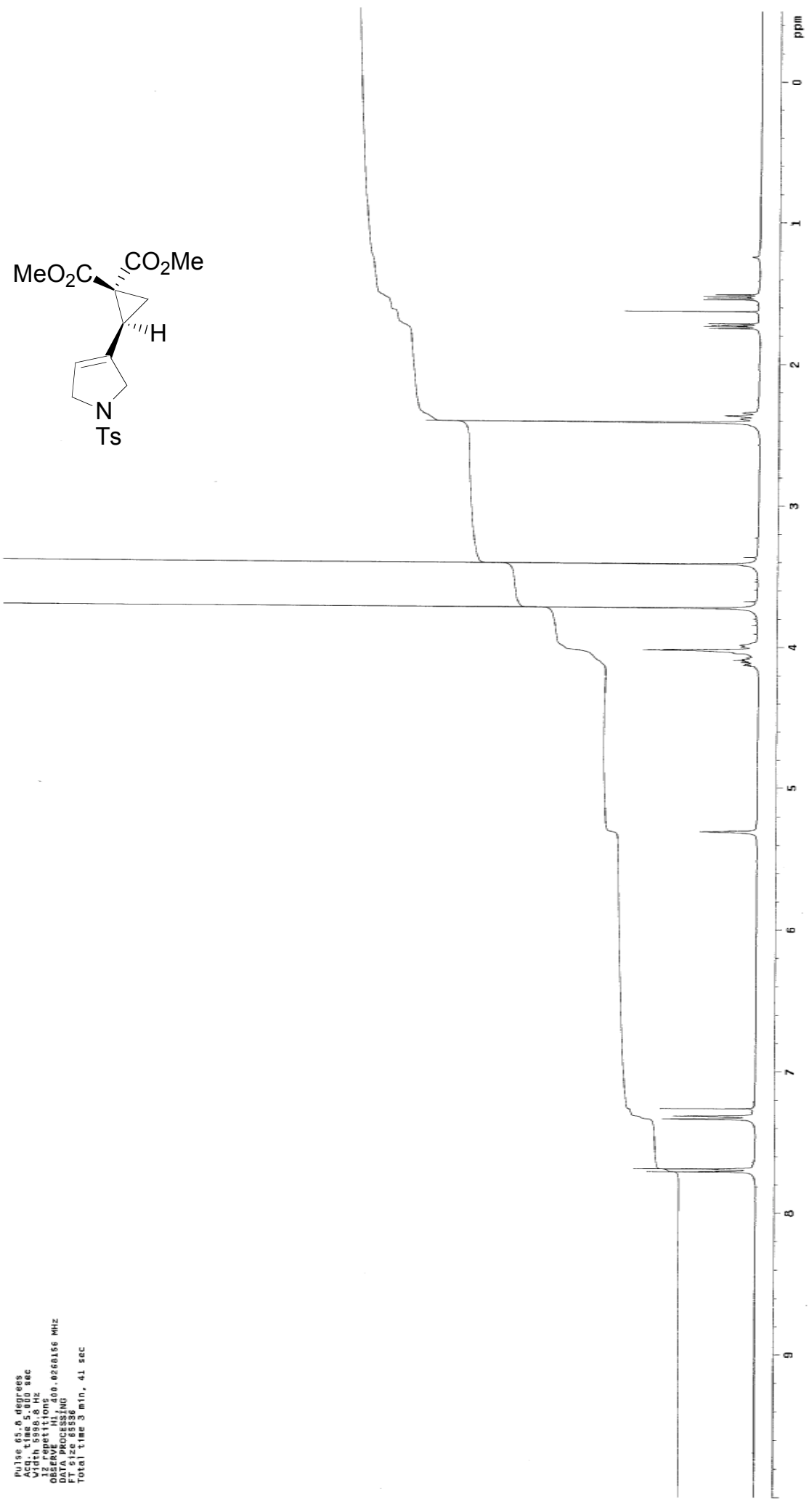
${ }^{1} \mathrm{H}$ NMR of $11 \mathrm{a}$.

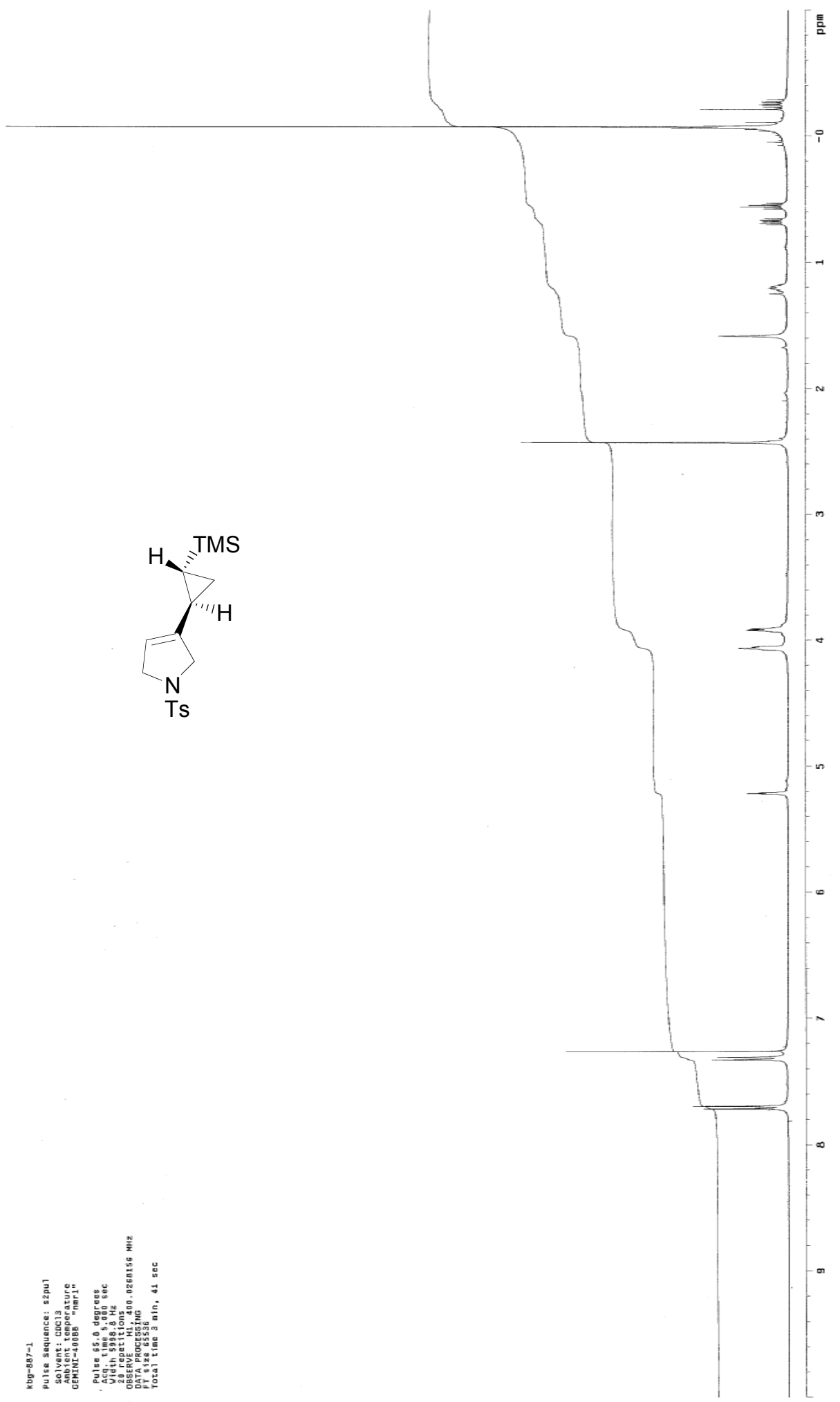


${ }^{1} \mathrm{H}$ NMR of $11 \mathrm{~b}$

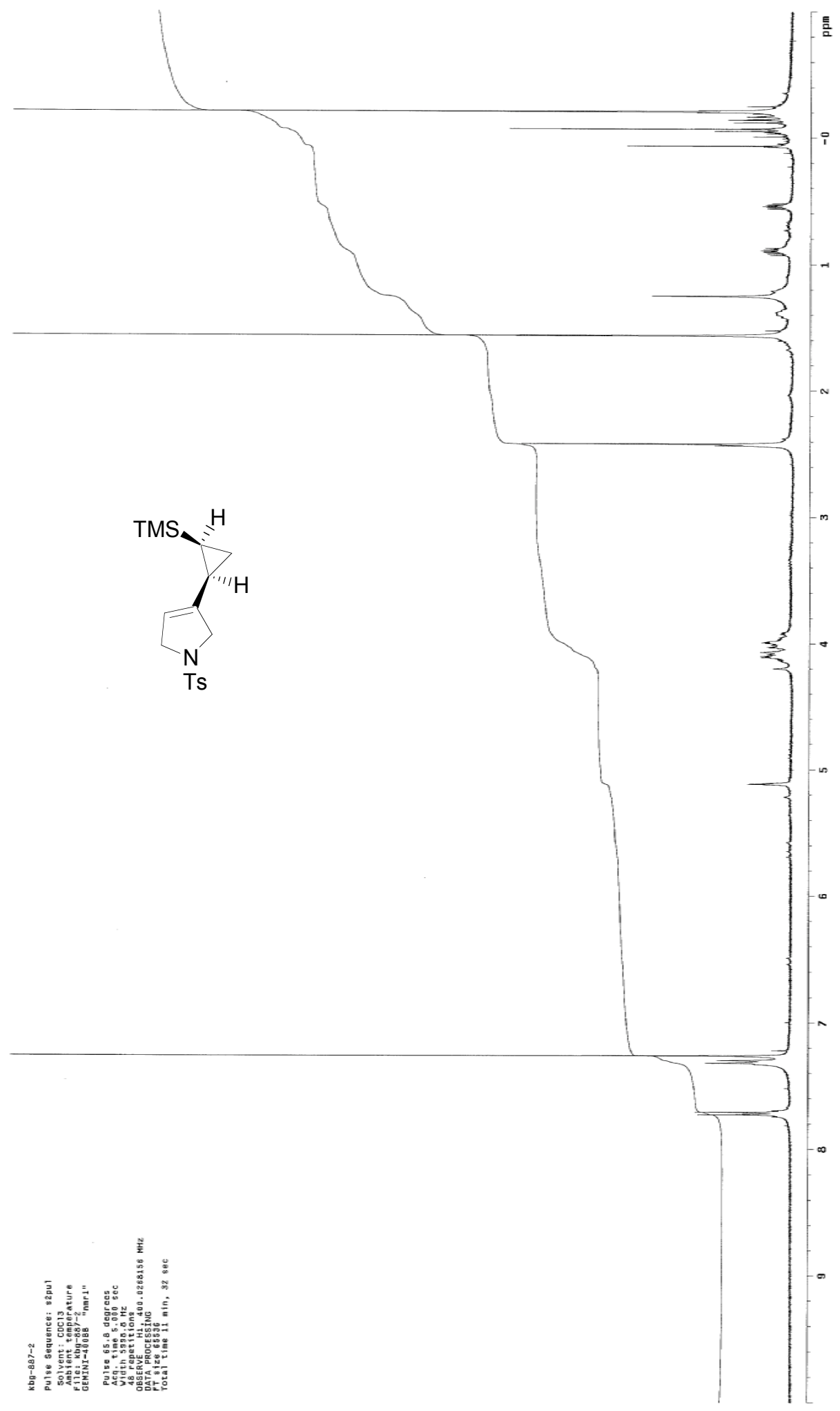


${ }^{1} \mathrm{H}$ NMR of $13 \mathbf{a}$
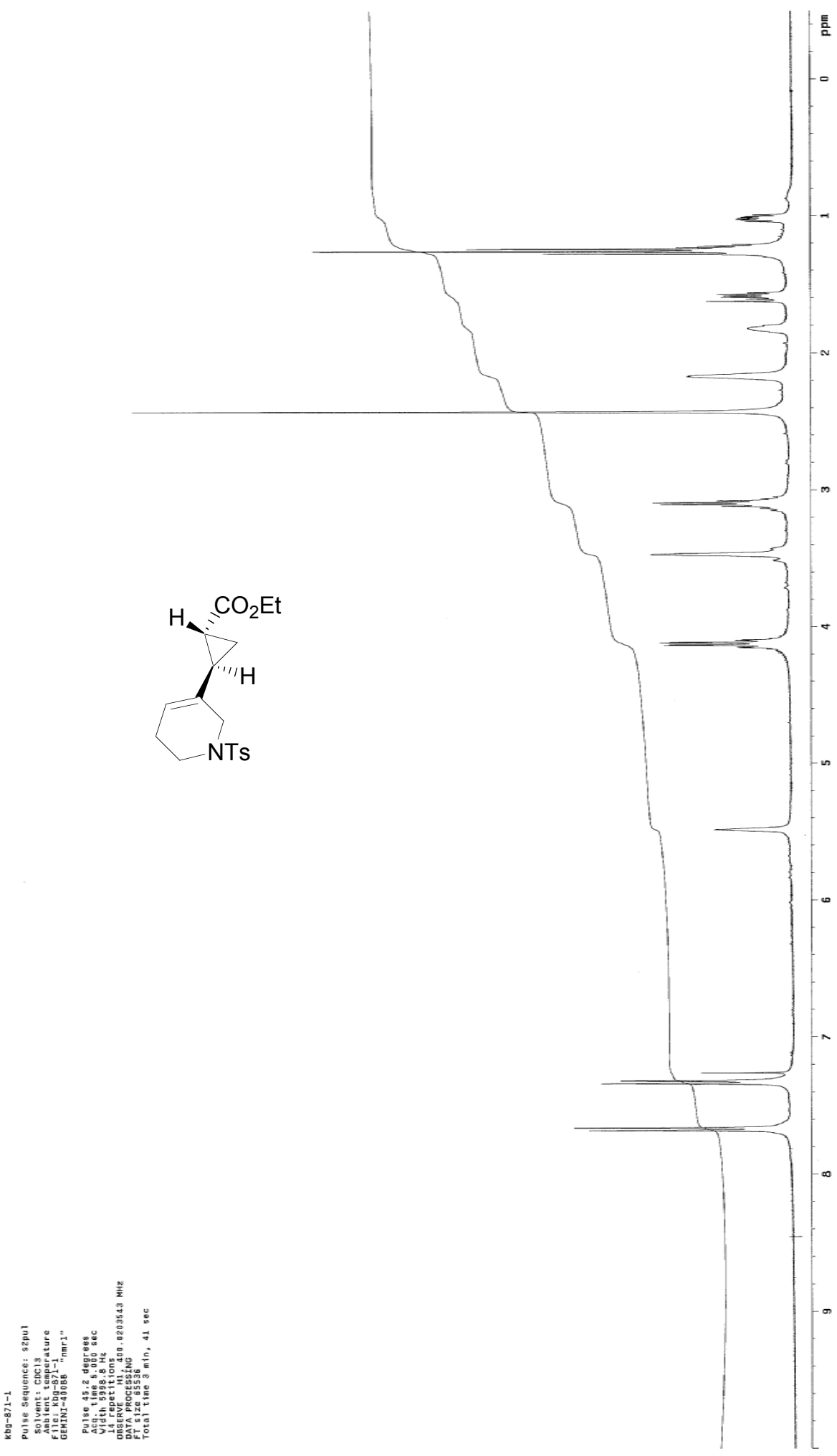
${ }^{1} \mathrm{H}$ NMR of $13 b$

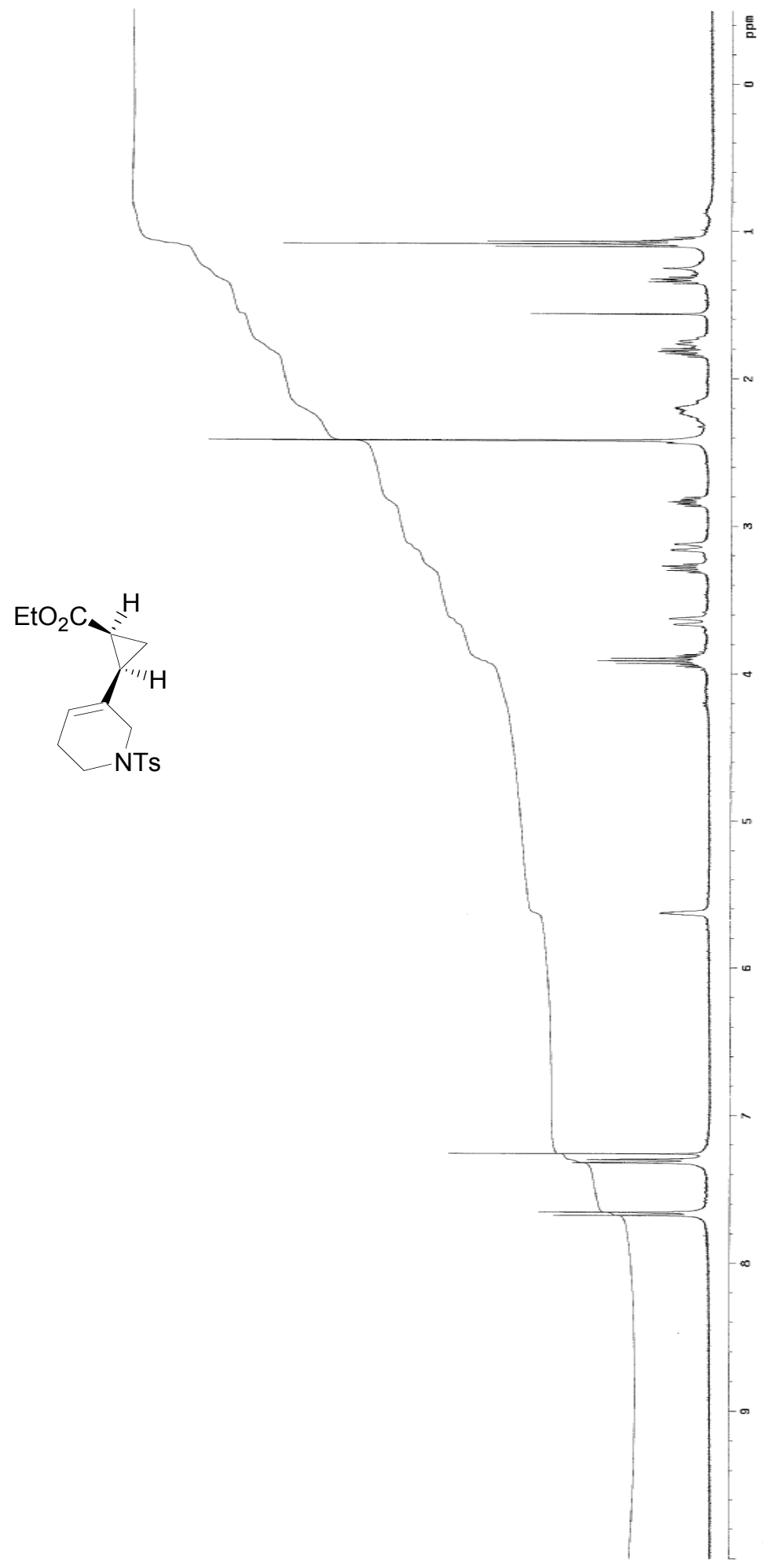


${ }^{1} \mathrm{H}$ NMR of $15 \mathbf{a}$

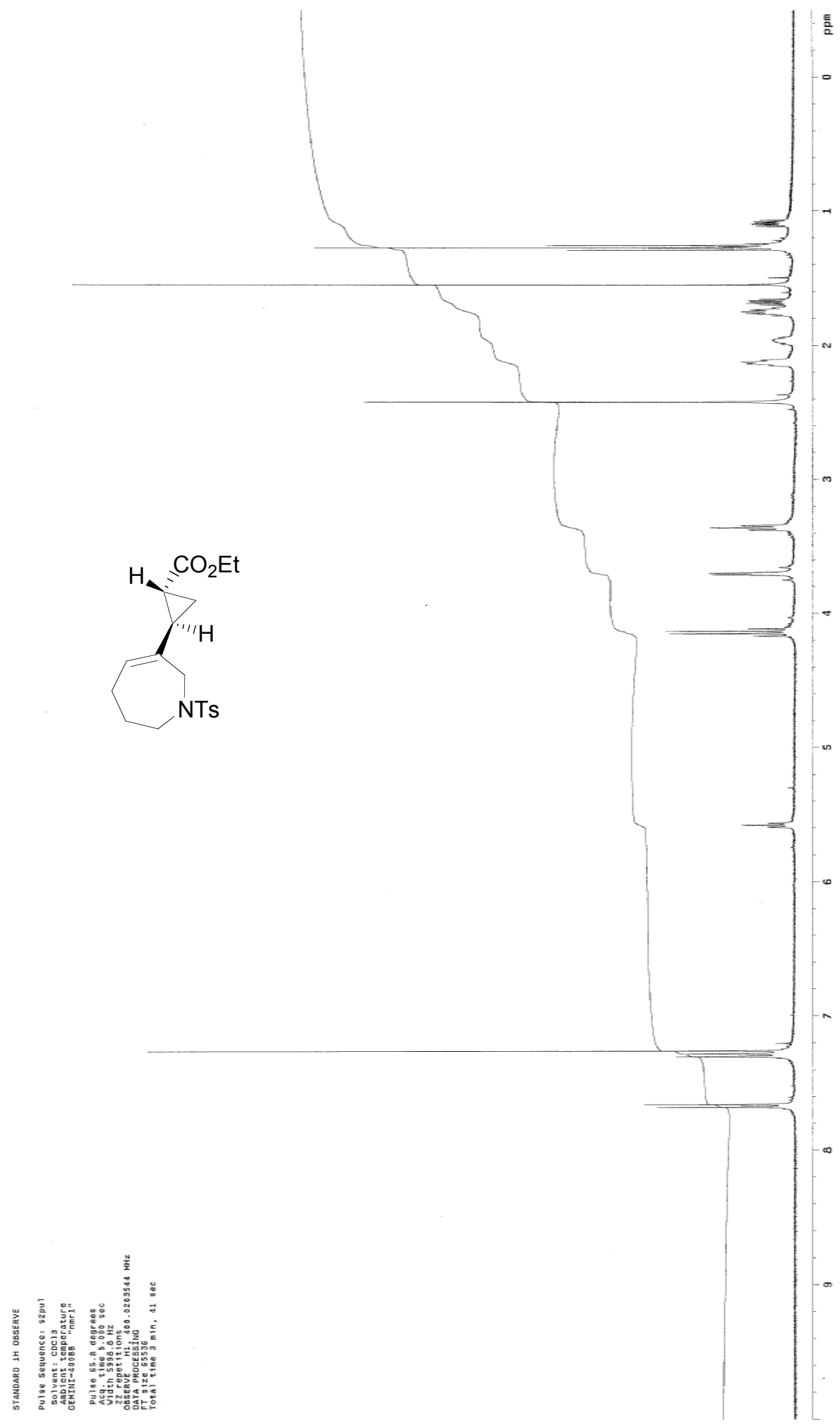


${ }^{1} \mathrm{H}$ NMR of $15 b$

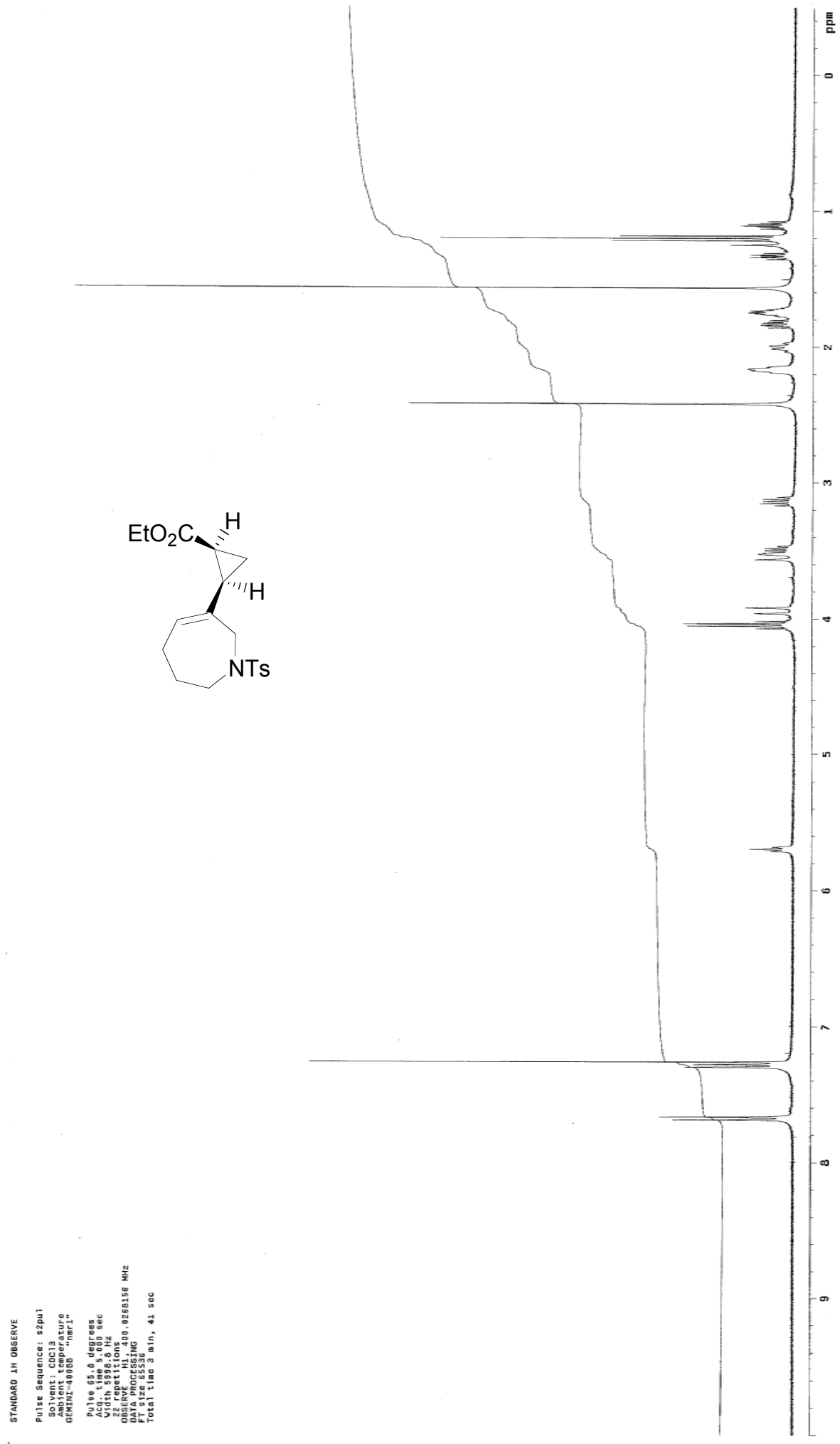


${ }^{1} \mathrm{H}$ NMR of $17 a$

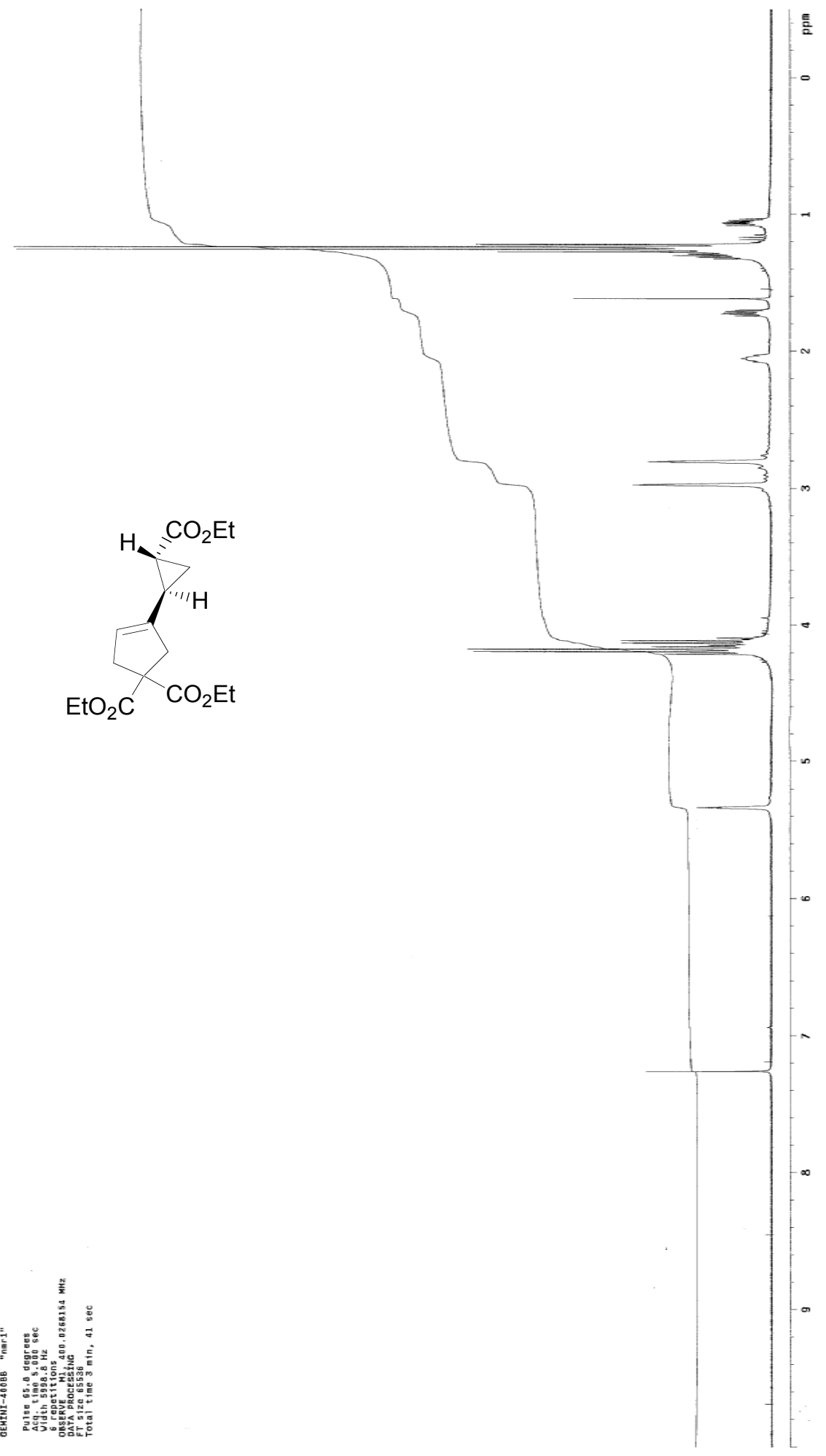


${ }^{1} \mathrm{H}$ NMR of $17 \mathbf{b}$
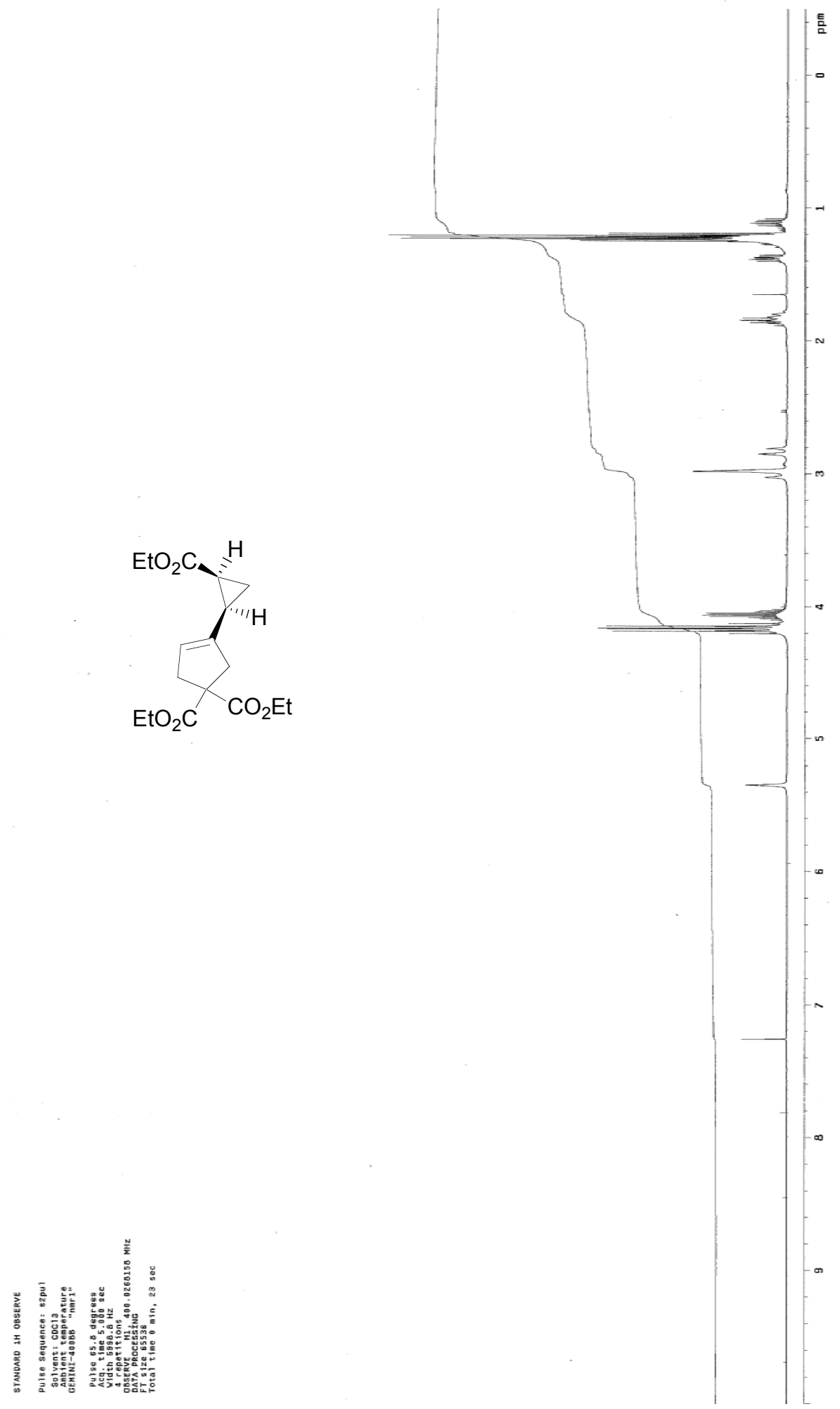
${ }^{1} \mathrm{H}$ NMR of $18 \mathbf{a}$
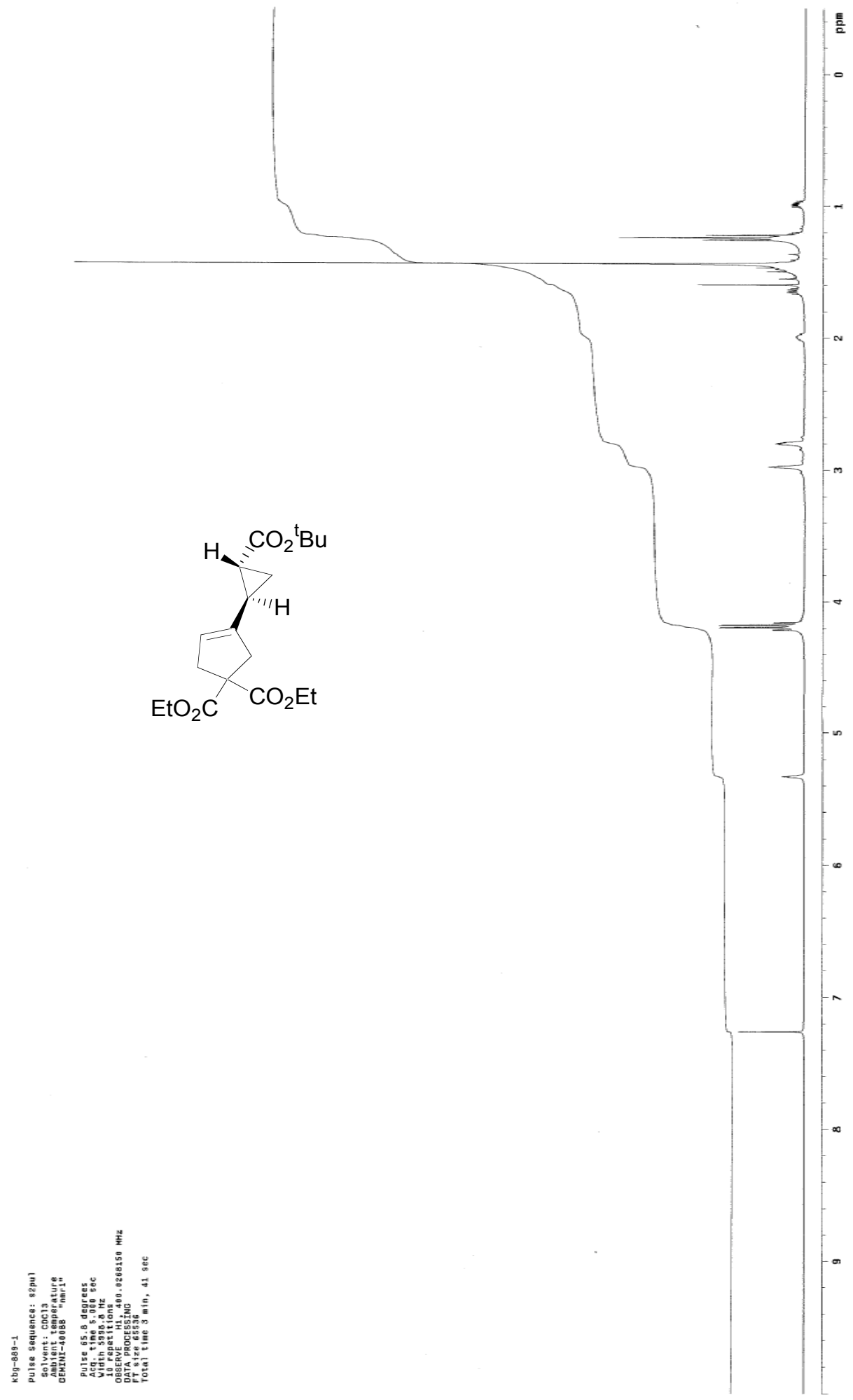
${ }^{1} \mathrm{H}$ NMR of $18 b$

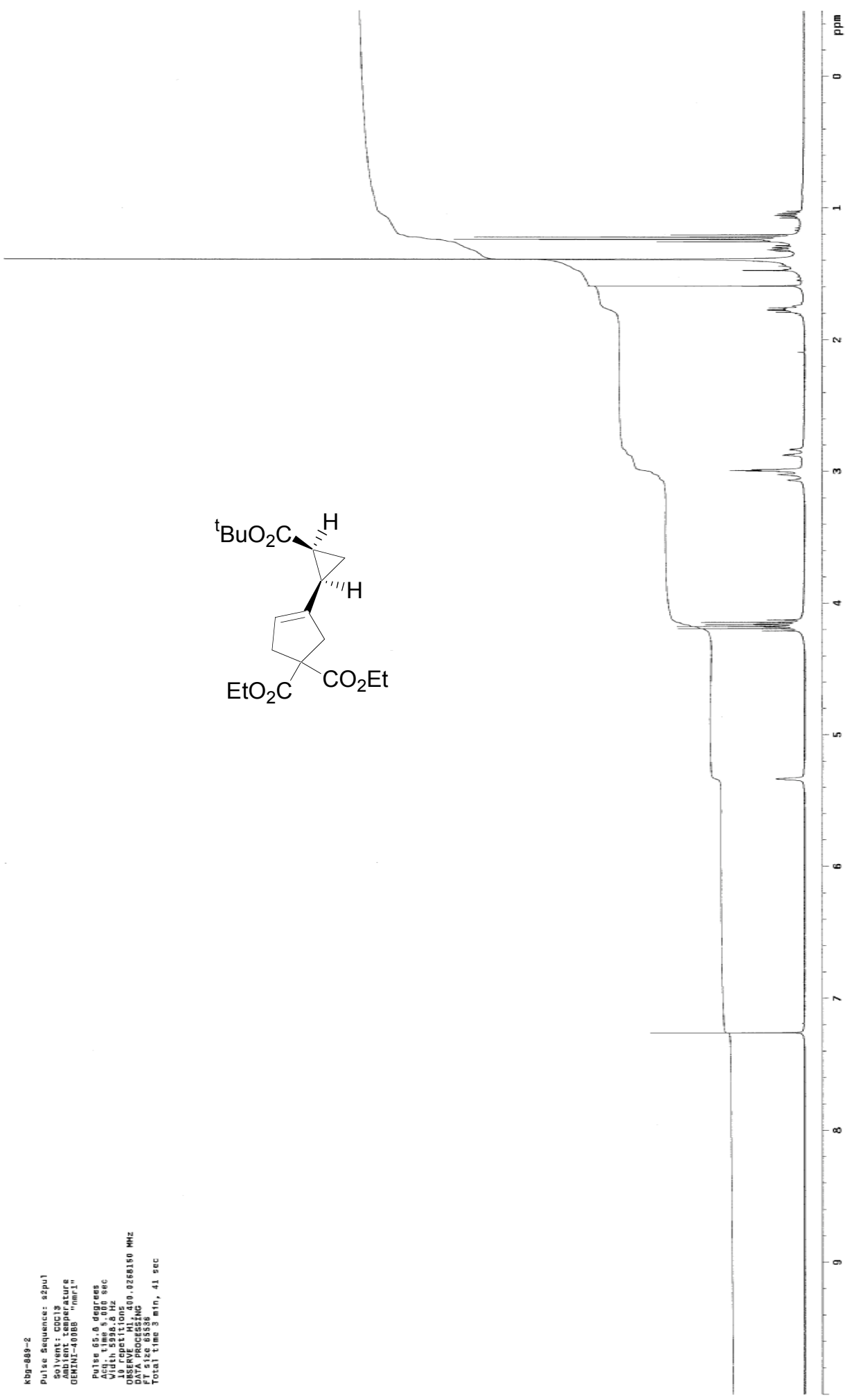


${ }^{1} \mathrm{H}$ NMR of $20 \mathrm{a}$.

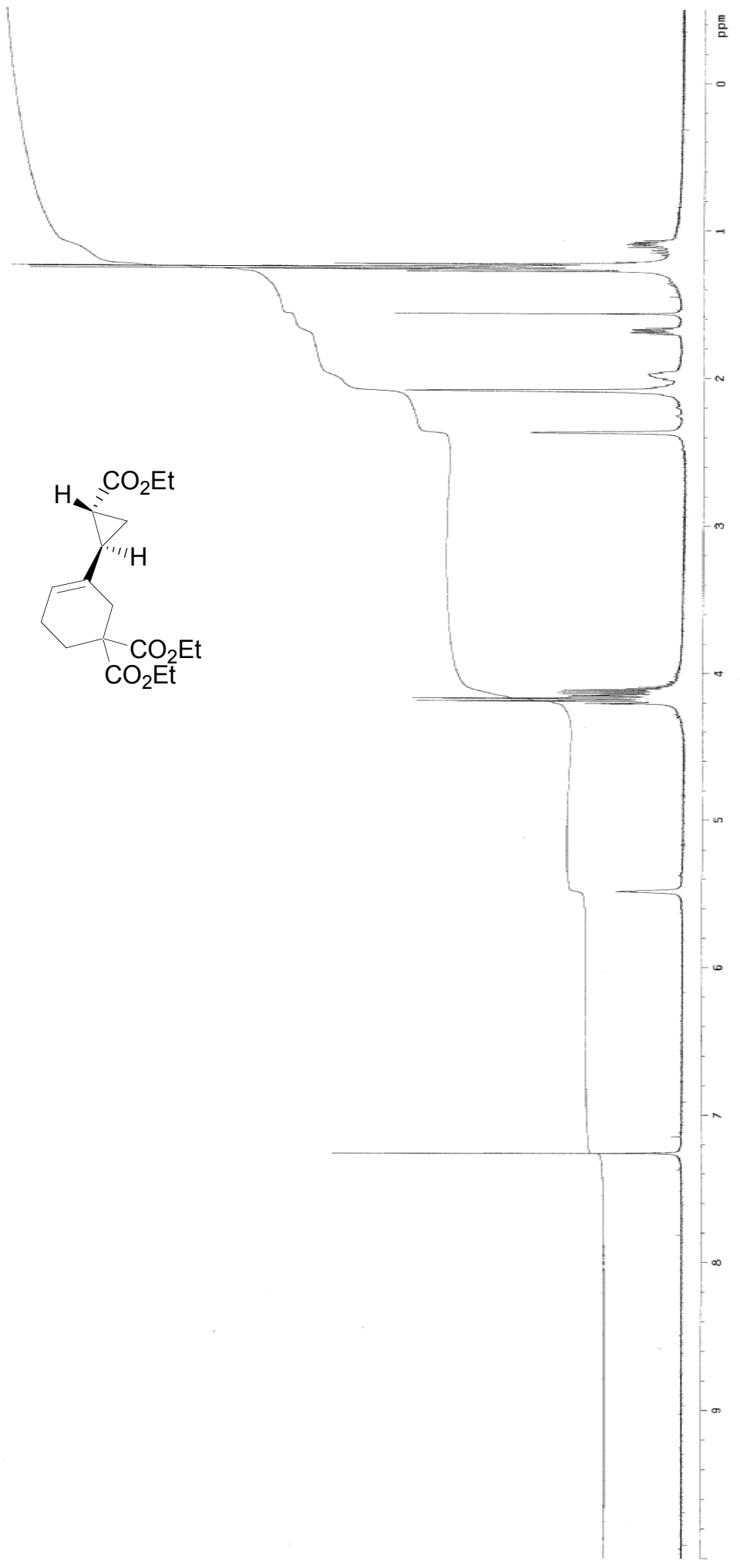


${ }^{1} \mathrm{H}$ NMR of $20 \mathrm{~b}$

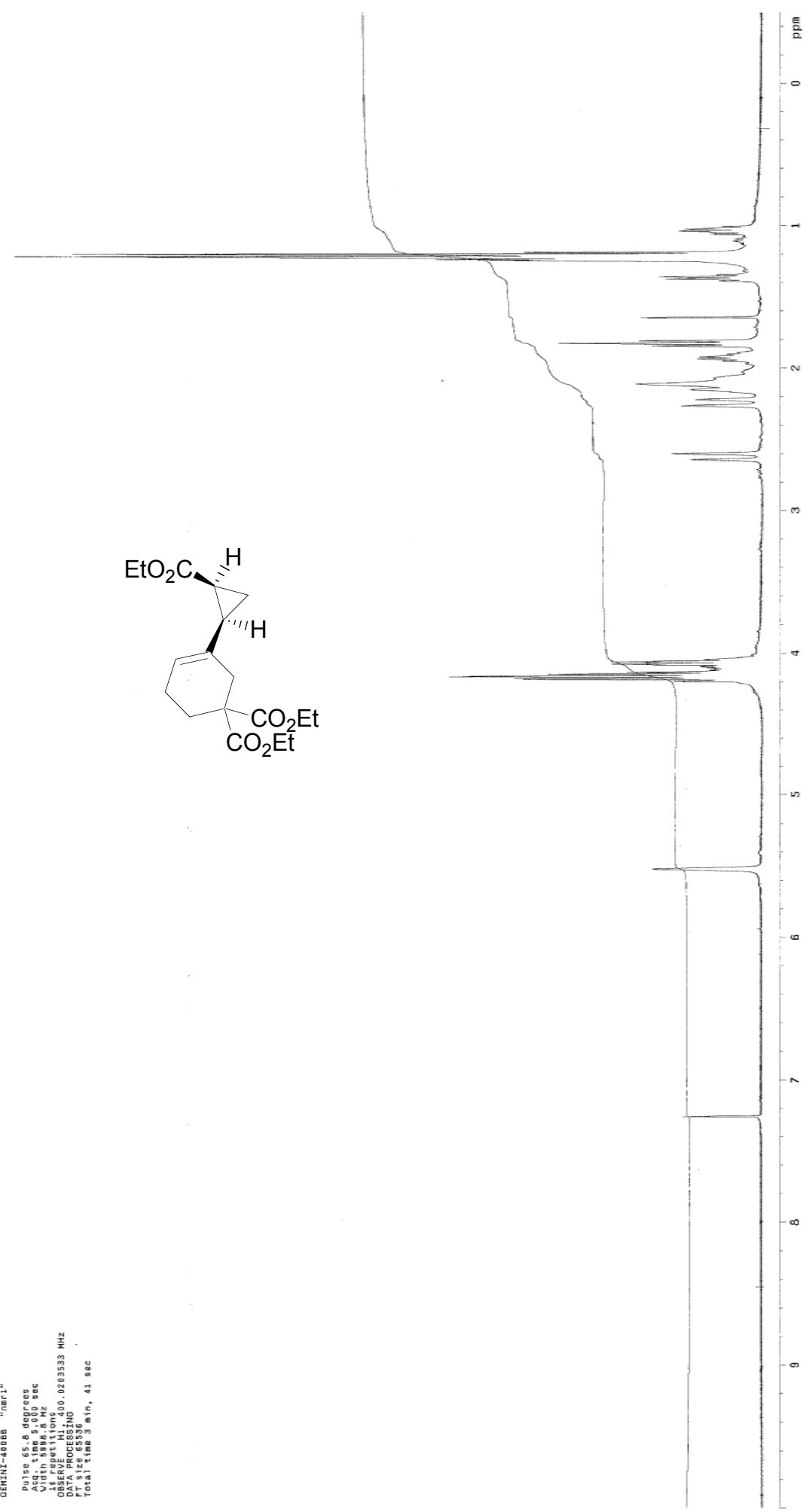


${ }^{1} \mathrm{H}$ NMR of $22 \mathrm{a}$.
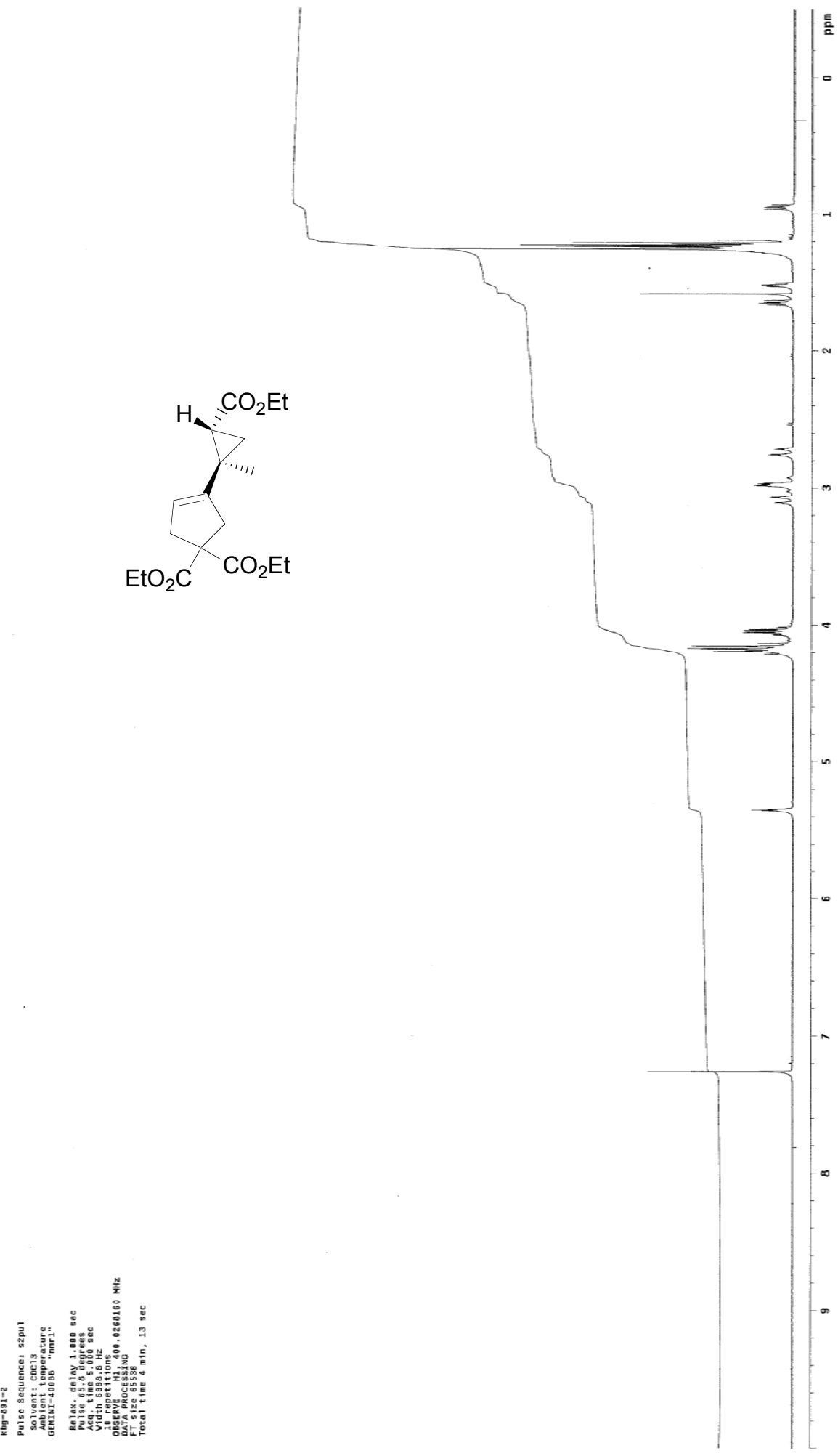
${ }^{1} \mathrm{H}$ NMR of $\mathbf{2 2 b}$
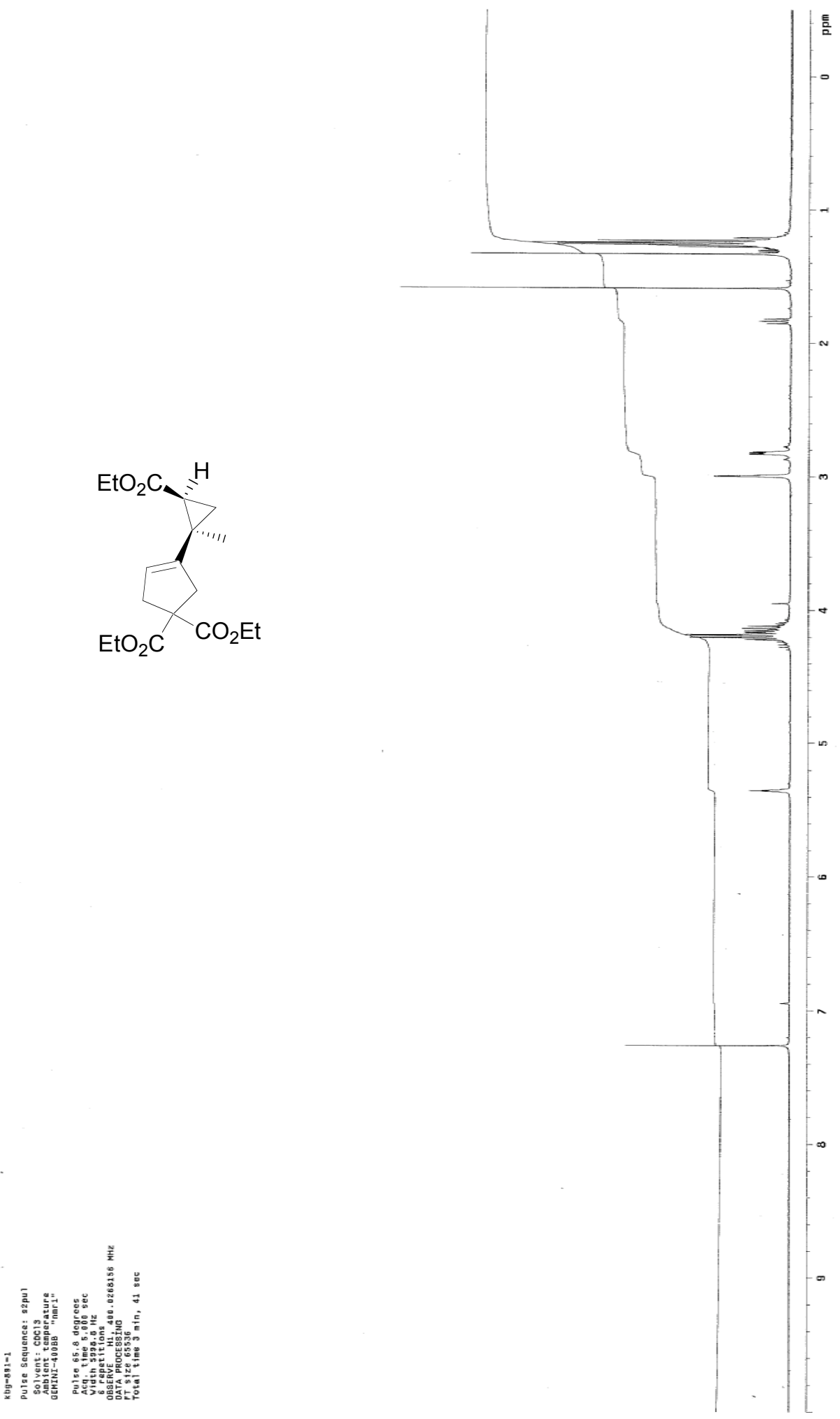